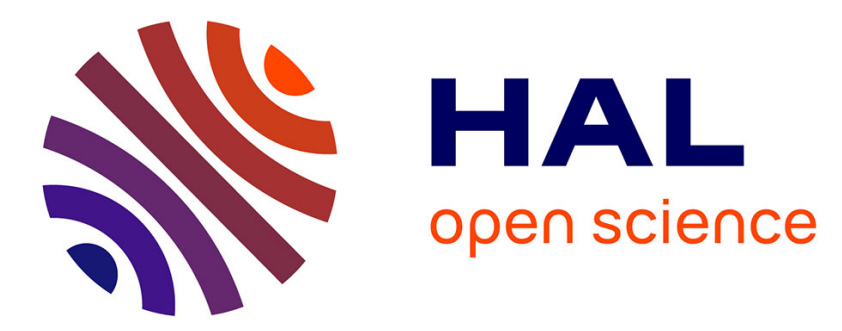

\title{
Goal-oriented adaptivity using unconventional error representations for the multidimensional Helmholtz equation
}

Vincent Darrigrand, Ángel Rodríguez-Rozas, Ignacio Muga, David Pardo, Albert Romkes, Serge Prudhomme

\section{To cite this version:}

Vincent Darrigrand, Ángel Rodríguez-Rozas, Ignacio Muga, David Pardo, Albert Romkes, et al.. Goaloriented adaptivity using unconventional error representations for the multidimensional Helmholtz equation. International Journal for Numerical Methods in Engineering, 2018, 113 (1), pp.22 - 42. 10.1002/nme.5601 . hal-01691495

\section{HAL Id: hal-01691495 \\ https://hal.science/hal-01691495}

Submitted on 4 Jan 2021

HAL is a multi-disciplinary open access archive for the deposit and dissemination of scientific research documents, whether they are published or not. The documents may come from teaching and research institutions in France or abroad, or from public or private research centers.
L'archive ouverte pluridisciplinaire HAL, est destinée au dépôt et à la diffusion de documents scientifiques de niveau recherche, publiés ou non, émanant des établissements d'enseignement et de recherche français ou étrangers, des laboratoires publics ou privés. 


\title{
Goal-oriented adaptivity using unconventional error representations for the multi-dimensional Helmholtz equation* ${ }^{* \dagger}$
}

\author{
Vincent Darrigrand ${ }^{1,2} ;$ Ángel Rodríguez-Rozas ${ }^{1,3}$, Ignacio Muga ${ }^{4}$, David Pardo ${ }^{1,3,5}$, \\ Albert Romkes ${ }^{6}$, and Serge Prudhomme ${ }^{7}$ \\ ${ }^{1}$ University of the Basque Country (UPV-EHU), Leioa, Spain \\ 2 Project-Team INRIA Magique-3D, INRIA Bordeaux-Sud Ouest, France. \\ ${ }^{3}$ Basque Center for Applied Mathematics (BCAM), Bilbao, Spain. \\ ${ }^{4}$ Pontificia Universidad Católica de Valparaíso, Chile. \\ ${ }^{5}$ Ikerbasque, Bilbao, Spain. \\ ${ }^{6}$ South Dakota School of Mines and Technology, Rapid City, USA \\ ${ }^{7}$ École Polytechnique de Montréal, Canada
}

\begin{abstract}
In Goal-Oriented Adaptivity (GOA), the error in the Quantity of Interest (QoI) is represented using the error functions of the direct and adjoint problems. This error representation is subsequently bounded above by element-wise error indicators that are used to drive optimal refinements. In this work, we propose to replace, in the error representation, the adjoint problem by an alternative operator. The main advantage of the proposed approach is that, when judiciously selecting such alternative operator, the corresponding upper bound of the error representation becomes sharper, leading to a more efficient GOA.

While the method can be applied to a variety of problems, we focus here on two- and threedimensional (2D and 3D) Helmholtz problems. We show via extensive numerical experimentation that the upper bounds provided by the alternative error representations are sharper than the classical ones and lead to a more robust $p$-adaptive process. We also provide guidelines for finding operators delivering sharp error representation upper bounds. We further extend the results to a convectiondominated diffusion problem as well as to problems with discontinuous material coefficients. Finally, we consider a sonic Logging-While-Drilling (LWD) problem to illustrate the applicability of the proposed method.
\end{abstract}

keywords Goal-Oriented Adaptivity, Finite Element Methods, Error Representation, Helmholtz Equation

\section{Introduction}

To obtain highly accurate finite element solutions, one often requires meshes that contain a large number of Degrees of Freedom (DoF). Since computational resources are limited, it is customary to build discretizations that require the smallest possible number of DoF to achieve a given tolerance error. As a

${ }^{*}$ This is the peer-reviewed version of the following article: Darrigrand, V, Rodríguez-Rozas, Á, Muga, I, Pardo, D, Romkes, A, Prudhomme, S. Goal-oriented adaptivity using unconventional error representations for the multidimensional Helmholtz equation. Int J Numer Meth Engng. 2018; 113: 22- 42., which has been published in final form at http: $/ / \mathrm{dx}$.doi.org/10.1002/nme.5601. This article may be used for non-commercial purposes in accordance with Wiley Terms and Conditions for Use of Self-Archived Versions.

†V. Darrigrand, Á. Rodríguez-Rozas and D. Pardo were partially funded by the Projects of the Spanish Ministry of Economy and Competitiveness with reference MTM2013-40824-P, MTM2016-76329-R (AEI/FEDER, EU), MTM201681697-ERC and the Basque Government Consolidated Research Group Grant IT649-13 on "Mathematical Modeling, Simulation, and Industrial Applications (M2SI)". Á. Rodríguez-Rozas and D. Pardo were also partially funded by the BCAM "Severo Ochoa" accreditation of excellence SEV-2013-0323 and the Basque Government through the BERC 2014-2017 program. Á. Rodríguez-Rozas acknowledges support from Spanish Ministry under Grant No. FPDI- 2013-17098. I. Muga was partially funded by the FONDECYT project 1160774. The first four authors were also partially funded by the European Union's Horizon 2020, research and innovation program under the Marie Sklodowska-Curie grant agreement No 644202. Serge Prudhomme is grateful for the support by a Discovery Grant from the Natural Sciences and Engineering Research Council of Canada.

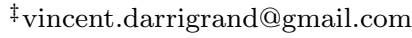


result, mesh-adaptive finite element algorithms arose. They were first developed to minimize the energy norm of the error per added DoF (see e.g. [1, 2]). However, in many engineering applications, one is interested in a specific Quantity of Interest (QoI). In these cases, energy-norm driven self-adaptive algorithms often fail to provide the required accuracy in the QoI using limited computational resources. For instance, Pardo et al. [3, 4] showed some electromagnetic applications in which an energy-norm adaptive algorithm reduces the energy-norm error to a level below $0.01 \%$, while the relative error in the QoI still remains above $15 \%$. In order to construct meshes that minimize the number of DoF while providing the required accuracy of the solution in a given QoI, the so-called Goal-Oriented Adaptivity (GOA) emerged.

There exist numerous engineering applications that motivate the use of GOA, including electromagnetics $[5,4,6,7,8]$, structural problems and visco-elasticity $[9,10,11,12,13]$, fluid-structure interactions $[14,15,16]$, and control theory $[17,18,19]$. Apart from these applications, convergence properties of GOA have also been recently studied in [20, 21, 22, 23, 24].

The origin of the GOA is in the works of Rannacher et al. $[25,26,27]$ followed by the works of Peraire, Patera et al. [28, 29, 30, 31, 32,33] on a posteriori error estimates of the error in the quantity of interest. The works of Prudhomme and Oden [34, 35, 36, 37] formulate the goal-oriented error estimation procedure based on representing the error in the QoI in terms of global functions defined over the entire computational domain. This error representation is subsequently bounded by the sum of local indicators that are used for the adaptive process.

In this work, we start with the methodology presented in [34], referred to as the classical goaloriented method. It employs the dual residual to derive upper bounds of the error in the QoI, and thus, indicators for the adaptive process. However, we depart from the classical approach by introducing an alternative dual operator for the representation of the error in the QoI, so the corresponding error bounds (indicators) become sharper than the classical ones. This new methodology generalizes the classical one. In particular, when the alternative dual operator coincides with the adjoint operator, we recover the error (upper) bounds of the classical goal-oriented method.

The main contribution in this work is the extension of the 1D results shown in [38] to the multidimensional case. In 1D (see [38]), a convergent $p$-adaptive algorithm was obtained either: (a) by combining the classical indicators and the Projection Based Interpolation (PBI) [39, 40, 41], or (b) by using the alternative indicators (with or without PBI). This result is extended here to the 2D and 3D cases and applied to Helmholtz and convection-dominated diffusion problems for continuous and discontinuous coefficients. We also show that finding in general the operator that provides the sharpest possible upper bounds is prohibitively expensive, and we provide a feasible alternative operator that delivers quite sharp upper bounds. A sonic logging-while-drilling (LWD) problem illustrates the applicability of the proposed method.

The remainder of the paper is organized as follows. In section 2, we define the model Helmholtz problem used in this investigation. In section 3, we recall the alternative method developed in [38], and we address the issue of finding the operator that provides the sharpest error upper bounds. In section 4, we present the $p$-adaptive algorithm and the software we have developed for the numerical computations. In section 5 , we analyze multiple 2D and 3D numerical results. We draw the main conclusions in section 6. This paper also contains three appendices describing additional numerical experiments about: (a) a convection-dominated diffusion problem (see Appendix A), (b) a problem with discontinuous material coefficients (see Appendix B), and (c) a geophysical borehole sonic LWD application (see Appendix C).

\section{Model problem}

\subsection{Definitions}

Given a domain $D \subset \mathbb{R}^{n}, \mathbb{H}(D)$ denotes a Hilbert space of functions defined over $D$, endowed with the norm $\|\cdot\|_{\mathbb{H}(D)}$.

Let $\Omega \subset \mathbb{R}^{n}$ be the physical domain of our problem of interest. Let $\mathcal{T}$ be a partition of $\Omega$ into open elements $K$ such that $\bar{\Omega}=\bigcup_{K \in \mathcal{T}} \bar{K}$.

We work with a Hilbert space $\mathbb{H}(\Omega)$ having the following property: If $w \in \mathbb{H}(\Omega)$, then its restriction $w_{K}$ to any open element $K \in \mathcal{T}$ satisfies $w_{K} \in \mathbb{H}(K)$. We define the restriction $R_{K}: \mathbb{H}(\Omega) \rightarrow \mathbb{H}(K)$ such that $R_{k}(v)=v_{k}, \forall v \in \mathbb{H}(\Omega)$. From now on, we will use the simplified notation $\mathbb{H}:=\mathbb{H}(\Omega)$ and $\mathbb{H}_{K}:=\mathbb{H}(K)$.

A bounded linear operator $B \in \mathcal{L}\left(\mathbb{H}, \mathbb{H}^{*}\right)$ is said to be localizable if, for any $K \in \mathcal{T}$, there exists 
$B_{K} \in \mathcal{L}\left(\mathbb{H}_{K}, \mathbb{H}_{K}^{*}\right)$ such that:

$$
\langle B w, v\rangle_{\mathbb{H}^{*}, \mathbb{H}}=\sum_{K \in \mathcal{T}}\left\langle B_{K} \circ R_{K} w, R_{K} v\right\rangle_{\mathbb{H}_{K}^{*}, \mathbb{H}_{K}}=\sum_{K \in \mathcal{T}}\left\langle B_{K} w_{K}, v_{K}\right\rangle_{\mathbb{H}_{K}^{*}, \mathbb{H}_{K}} .
$$

In other words, $B=\sum_{K \in \mathcal{T}} R_{K}^{*} \circ B_{K} \circ R_{K}$.

Remark: It is straightforward to show that if $B: \mathbb{H} \rightarrow \mathbb{H}^{*}$ is localizable, then the formal adjoint operator $B^{*}: \mathbb{H} \rightarrow \mathbb{H}^{*}$ is also localizable and $B^{*}=\sum_{K} R_{K}^{*} \circ B_{K}^{*} \circ R_{K}$.

\subsection{Helmholtz equation}

We select the Helmholtz equation for its wide use in wave propagation problems. Specifically, we consider the following problem with mixed boundary conditions: for $\Omega=(0,1)^{d} \subset \mathbb{R}^{d}$, with boundary $\partial \Omega$ partitioned into two parts $\Gamma_{I}$ and $\Gamma_{D}$ such that $\bar{\Gamma}_{D} \cup \bar{\Gamma}_{I}=\partial \Omega$, and $\stackrel{\circ}{\Gamma}_{D} \bigcap \stackrel{\circ}{\Gamma}_{I}=\emptyset$,

$$
\begin{aligned}
& \text { Find } u \text { such that, given } k>0, \\
& \qquad \begin{cases}-\Delta u-k^{2} u=1 & \text { in } \Omega, \\
u=0 & \text { on } \Gamma_{D}, \\
\partial_{n} u+i k u=0 & \text { on } \Gamma_{I},\end{cases}
\end{aligned}
$$

We set $\mathbb{H}:=\left\{u \in H^{1}(\Omega), u_{\mid \Gamma_{D}}=0\right\}$ and $\langle\cdot, \cdot\rangle_{L^{2}}$ the standard $L^{2}$ sesquilinear product. We define the QoI $l$ as the linear functional in $\mathbb{H}^{*}$ corresponding to the integral of $w \in \mathbb{H}$ on a portion $\Gamma_{\mathrm{QoI}}$ of the boundary $\Gamma_{I}$ :

$$
\langle l, w\rangle_{\mathbb{H}^{*}, \mathbb{H}}=\langle 1, w\rangle_{L^{2}\left(\Gamma_{\mathrm{QoI}}\right)} \quad \forall w \in \mathbb{H} .
$$

Operator $B \in \mathcal{L}\left(\mathbb{H}, \mathbb{H}^{*}\right)$ associated with above problem is defined as follows,

$$
\langle B w, z\rangle_{\mathbb{H}^{*}, \mathbb{H}}=\langle\nabla w, \nabla z\rangle_{L^{2}(\Omega)}-k^{2}\langle w, z\rangle_{L^{2}(\Omega)}+i k\langle w, z\rangle_{L^{2}\left(\Gamma_{I}\right)}, \quad \forall w, z \in \mathbb{H} .
$$

Notice that the above problem is numerically unstable for high wavenumbers [42, 43, 44, 45, 46, 47, 48, $49,50]$.

For the $2 \mathrm{D}$ case, $d=2$, the boundaries are set as

$$
\begin{aligned}
\Gamma_{D} & :=([0,1] \times\{0\}) \bigcup(\{0\} \times[0,1]), \\
\Gamma_{I} & :=([0,1] \times\{1\}) \bigcup(\{1\} \times[0,1]), \\
\Gamma_{\mathrm{QoI}} & :=\{1\} \times(0.75,1),
\end{aligned}
$$

as illustrated in Figure 1.

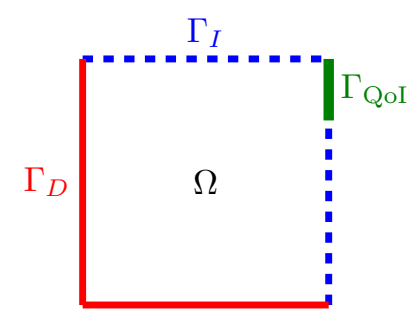

Figure 1: Computational domain in 2D

For the 3D case, $d=3$ (see Figure 2), we set the boundaries as follows: A Dirichlet boundary condition is prescribed on the three faces whose intersection is $(0,0,0)$ and an impedance boundary condition is 
imposed on the three faces whose intersection is $(1,1,1)$.

$$
\begin{aligned}
& \Gamma_{D}:=([0,1] \times[0,1] \times\{0\}) \bigcup([0,1] \times\{0\} \times[0,1]) \\
& \bigcup(\{0\} \times[0,1] \times[0,1]) \\
& \Gamma_{I}:=([0,1] \times[0,1] \times\{1\}) \bigcup([0,1] \times\{1\} \times[0,1]) \\
& \bigcup(\{1\} \times[0,1] \times[0,1]) \\
& \Gamma_{\text {QoI }}:=([0.75,1] \times[0.75,1] \times\{1\}) \bigcup([0.75,1] \times\{1\} \times[0.75,1]) \\
& \bigcup(\{1\} \times[0.75,1] \times[0.75,1]) .
\end{aligned}
$$

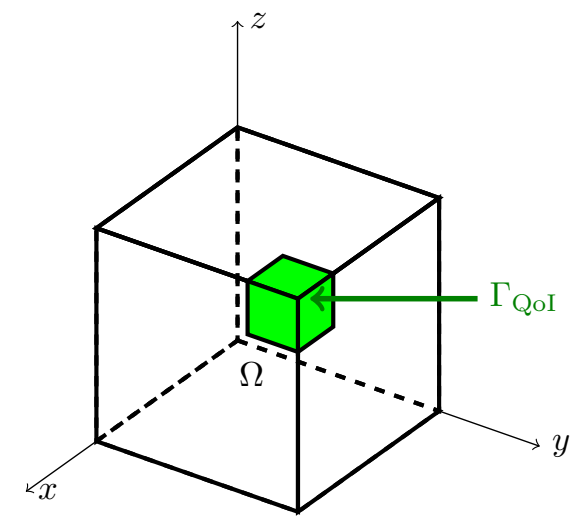

Figure 2: Computational domain for our 3D Helmholtz problem

\section{Error representations for Goal Oriented Adaptivity}

In this Section, we briefly recall the method described in [38] and reformulate it in terms of operators.

\subsection{Classical goal-oriented formulation}

Consider a loading form $f \in \mathbb{H}^{*}$ and a QoI $l \in \mathbb{H}^{*}$. Let $\mathbb{H}_{h} \subset \mathbb{H}$ be a conforming finite element subspace associated with partition $\mathcal{T}$. Let $B \in \mathcal{L}\left(\mathbb{H}, \mathbb{H}^{*}\right)$ be a localizable operator such that there exists a unique solution for each of both continuous and discrete direct and dual problems:

$$
\begin{aligned}
& \text { Find } u \in \mathbb{H} \text { and } u_{h} \in \mathbb{H}_{h} \text { such that } \\
& \qquad \begin{array}{l}
B u=f \quad \text { in } \mathbb{H}^{*} \\
\left\langle B u_{h}, w_{h}\right\rangle_{\mathbb{H}^{*}, \mathbb{H}}=\left\langle f, w_{h}\right\rangle_{\mathbb{H}^{*}, \mathbb{H}}, \quad \forall w_{h} \in \mathbb{H}_{h} .
\end{array}
\end{aligned}
$$

and

Find $v \in \mathbb{H}$ and $v_{h} \in \mathbb{H}_{h}$ such that

$$
\begin{aligned}
& B^{*} v=l \quad \text { in } \mathbb{H}^{*} \\
& \left\langle B^{*} v_{h}, w_{h}\right\rangle_{\mathbb{H}^{*}, \mathbb{H}}=\left\langle l, w_{h}\right\rangle_{\mathbb{H}^{*}, \mathbb{H}}, \quad \forall w_{h} \in \mathbb{H}_{h} .
\end{aligned}
$$

The errors in the approximations of the direct and adjoint problems are defined as $e=u-u_{h}$ and $\varepsilon=v-v_{h}$, respectively. One can also represent these errors as solutions of the following variational problems: 
- Find $e \in \mathbb{H}$ such that

$$
B e=f-B u_{h}=: \mathcal{R}_{p}^{h}
$$

- Find $\varepsilon \in \mathbb{H}$ such that

$$
B^{*} \varepsilon=l-B^{*} v_{h}=: \mathcal{R}_{d}^{h}
$$

Functionals $\mathcal{R}_{p}^{h}, \mathcal{R}_{d}^{h} \in \mathbb{H}^{*}$ are known as the primal and dual residuals, respectively.

Evaluating (3.3) at $e$, using Galerkin's orthogonality and the localization property of $B$, we obtain

$$
\langle l, e\rangle_{\mathbb{H}^{*}, \mathbb{H}}=\left\langle\mathcal{R}_{d}^{h}, e\right\rangle_{\mathbb{H}^{*}, \mathbb{H}}=\left\langle B^{*} \varepsilon, e\right\rangle_{\mathbb{H}^{*}, \mathbb{H}}=\sum_{K \in \mathcal{T}}\left\langle B_{K}^{*} \varepsilon_{K}, e_{K}\right\rangle_{\mathbb{H}_{K}^{*}, \mathbb{H}_{K}}
$$

Thus, an upper bound of the error in the QoI is given as follows:

$$
\left|\langle l, e\rangle_{\mathbb{H}^{*}, \mathbb{H}}\right| \leqslant \sum_{K \in \mathcal{T}}\left|\left\langle B_{K}^{*} \varepsilon_{K}, e_{K}\right\rangle_{\mathbb{H}_{K}^{*}, \mathbb{H}_{K}}\right|=\sum_{K \in \mathcal{T}} \eta_{K}=: \eta_{\mathcal{T}}
$$

where $\eta_{K}:=\left|\left\langle B_{K}^{*} \varepsilon_{K}, e_{K}\right\rangle_{\mathbb{H}_{K}^{*}, \mathbb{H}_{K}}\right|$. This upper bound will be referred to as the classical bound.

\subsection{Alternative representations}

The key idea proposed here is to use alternative representations of the residuals $\mathcal{R}_{d}^{h}$ or $\mathcal{R}_{p}^{h}$. Let $\widetilde{B} \in$ $\mathcal{L}\left(\mathbb{H}, \mathbb{H}^{*}\right)$ be a localizable invertible operator. We define the alternative dual error representation as the solution of the linear equation:

$$
\begin{aligned}
& \text { Find } \widetilde{\varepsilon} \in \mathbb{H} \text { such that } \\
& \qquad \widetilde{B} \widetilde{\varepsilon}=\mathcal{R}_{d}^{h} .
\end{aligned}
$$

Analogously, we define the alternative primal error representation as the solution of the linear equation:

$$
\text { | Find } \widetilde{e} \in \mathbb{H} \text { such that } \quad \widetilde{B} \widetilde{e}=\mathcal{R}_{p}^{h} .
$$

For simplicity, we use the same operators for construction of the alternative direct and dual errors. However, it is possible to select different representations for each error.

Following the same procedure as in (3.4) and (3.5), we obtain the alternative bound of the error in the QoI

$$
\left|\langle l, e\rangle_{\mathbb{H}^{*}, \mathbb{H}}\right| \leqslant \sum_{K \in \mathcal{T}}\left|\left\langle\widetilde{B}_{K} \widetilde{\varepsilon}_{K}, e_{K}\right\rangle_{\mathbb{H}_{K}^{*}, \mathbb{H}_{K}}\right|=\sum_{K \in \mathcal{T}} \widetilde{\eta}_{K}=: \widetilde{\eta}_{\mathcal{T}}
$$

where $\widetilde{\eta}_{K}:=\left|\left\langle\widetilde{B}_{K} \widetilde{\varepsilon}_{K}, e_{K}\right\rangle_{\mathbb{H}_{K}^{*}, \mathbb{H}_{K}}\right|$. One can alternatively make use of the primal error representation $\widetilde{e}$ to obtain the bound:

$$
\left|\langle l, e\rangle_{\mathbb{H}^{*}, \mathbb{H}}\right| \leqslant \sum_{K \in \mathcal{T}}\left|\left\langle\widetilde{B}_{K} \widetilde{e}_{K}, \varepsilon_{K}\right\rangle_{\mathbb{H}_{K}^{*}, \mathbb{H}_{K}}\right| .
$$

Numerical results (see Figure 7b) show that upper bounds given by (3.7) and (3.8) are similar. Hence and for simplicity, in the following we will consider Eq. (3.7), disregarding Eq. (3.8).

The method presented here is indeed a generalization of the classical GOA, that is recovered by simply selecting $\widetilde{B}=B^{*}$ or $\widetilde{B}=B$.

An interesting case occurs when additionally each local counterpart $\widetilde{B}_{K}$ of $\widetilde{B}$ is self-adjoint and semi-positive definite. In that case, operator $\widetilde{B}_{K}$ defines a semi-inner product on $\mathbb{H}_{K}$ and we can take additional Cauchy-Schwarz inequalities on (3.7), i.e.,

$$
\widetilde{\eta}_{\mathcal{T}} \leqslant \sum_{K \in \mathcal{T}} \sqrt{\left\langle\widetilde{B}_{K} \widetilde{\varepsilon}_{K}, \widetilde{\varepsilon}_{K}\right\rangle_{\mathbb{H}_{K}^{*}, \mathbb{H}_{K}}} \sqrt{\left\langle\widetilde{B}_{K} e_{K}, e_{K}\right\rangle_{\mathbb{H}_{K}^{*}, \mathbb{H}_{K}}}
$$




\subsection{Optimal Alternative Operator}

The sharpest bound is obtained by an operator for which the triangle inequality in Equation (3.7) becomes an equality, namely:

$$
\left|\langle l, e\rangle_{\mathbb{H}^{*}, \mathbb{H}}\right|=\left|\sum_{K \in \mathcal{T}}\left\langle\widetilde{B}_{K} \widetilde{\varepsilon}_{K}, e_{K}\right\rangle_{\mathbb{H}_{K}^{*}, \mathbb{H}_{K}}\right|=\sum_{K \in \mathcal{T}}\left|\left\langle\widetilde{B}_{K} \widetilde{\varepsilon}_{K}, e_{K}\right\rangle_{\mathbb{H}_{K}^{*}, \mathbb{H}_{K}}\right| .
$$

This means that on each element $K \in \mathcal{T}$, the complex quantities $\left\langle\widetilde{B}_{K} \widetilde{\varepsilon}_{K}, e_{K}\right\rangle_{\mathbb{H}_{K}^{*}, \mathbb{H}_{K}}$ are sharing the same angle. It implies as well that each estimator needs to share the same angle of $\langle l, e\rangle_{\mathbb{H}^{*}, \mathbb{H}^{*}}$. To simplify, let us assume that they are all positive real numbers. Then, we have to find an operator $\widetilde{B}$ such that the element-wise application $\widetilde{B}_{K} \widetilde{\varepsilon}_{K} \in \mathbb{H}_{K}^{*}$ has to compensate the variations of $e_{K}$ in order to obtain a positive real number after integration. The consequence is that operator $\widetilde{B}=\sum_{K \in \mathcal{T}} R_{K}^{*} \circ \widetilde{B}_{K} \circ R_{K}$ has to be defined on each element according to $e_{K}$. It will probably occur that, if it exists, $\widetilde{B}$ will not be a conventional variational form, which will make much more complex the implementation of the method. Thus, in this work, rather than searching for the optimal operator, we shall concentrate on finding the best possible operator within a preset family via numerical experimentation.

\subsection{Selection of the Alternative Operator}

Let $B$ be the 2D Helmholtz operator defined by (2.1) with $k \in \mathbb{R}^{+}$, with a source term $f \in \mathbb{H}^{*}$, and a QoI $l \in \mathbb{H}^{*}$, as defined in Section 2. We set the wavenumber to $k=17 \pi$. We analyze the behavior of $\widetilde{\eta}_{\mathcal{T}}$ of Eq. 3.7 when varying the alternative operator $\widetilde{B}_{\alpha}$, for a given discretization $\mathcal{T}$, over the family $\mathcal{U}$ :

$$
\mathcal{U}:=\left\{\widetilde{B}_{\alpha}, \alpha \in \mathbb{C}\right\}
$$

where

$$
\left\langle\widetilde{B}_{\alpha} \cdot, \cdot\right\rangle_{\mathbb{H}^{*}, \mathbb{H}}=\langle\nabla \cdot, \nabla \cdot\rangle_{L^{2}(\Omega)}+\alpha\langle\cdot, \cdot\rangle_{L^{2}(\Omega)}+i \sqrt{|\alpha|}\langle\cdot, \cdot\rangle_{L^{2}\left(\Gamma_{I}\right)} .
$$

The boundary conditions for $\widetilde{B}_{\alpha}$ are selected to be the same as those of the original operator $B$.

In the following, we approximate space $\mathbb{H}$ by a finite element subspace richer than $\mathbb{H}_{h}$ by increasing uniformly the polynomial order of approximation by $\Delta p=2$. By an abuse of notation, we will denote the elements of $\mathbb{H}$ and their approximation in the richer space with the same symbols. Although in some cases the fine mesh should be finer in order to better estimate the error, the high regularity of the solutions of our model problems justify such choice of fine mesh. In any case, we emphasize that the focus of this work is on the error representations of a given QoI, and not on the efficiency of how to compute the error function itself.

Figures 3 and 4 show the evolution of $\widetilde{\eta}_{\mathcal{T}}$ with respect to $\alpha$ and along specific directions: in Figure 3 the parameter $\alpha$ is real, whereas in Figure 4 the parameter $\alpha$ is purely imaginary.

The sharpest upper bounds are obtained for $\alpha=0$ (Laplace operator) in most cases. When $\alpha$ is real (see Figure 3), the Laplace operator is not delivering exactly the sharpest bound, but it is very close to it.

Top panel of Figure 3 shows that for large $|\alpha|,\left(\geqslant 10^{5}\right)$, the behavior of the alternative operator is almost equivalent to that of the $L^{2}$-sesquilinear product. If $\alpha>0, \widetilde{B}_{\alpha}$ is self-adjoint and positive definite. For both cases $\left(\alpha \geqslant 10^{5}\right.$ and $\left.\alpha \leqslant-10^{5}\right)$, the alternative upper bounds are sharper than the classical ones. If $\alpha<0$, then we are dealing with a Helmholtz operator. To numerically resolve Equation (3.6), we need to satisfy the Nyquist rate. When $\alpha<-k^{2}$ (the red area), the Nyquist rate criterion is compromised, and the numerical resolution is untrustworthy. Thus, we restrict the analysis to $\alpha \in\left[-k^{2}, 0\right]$. The second graph (Figure 3, middle panel) zooms on this area. We observe that bound $\widetilde{\eta}_{\mathcal{T}}$ is slightly oscillating as $\alpha$ becomes more negative, probably because the number of DoF per wavelength is getting smaller and the dispersion effect is stronger. The almost flat area for $\alpha>-800$ is rescaled in Figure 3 (bottom panel) in order to determine whether or not the Laplacian is the operator that provides the sharpest upper bound. We observe that the minimum is not reached for $\alpha=0$, but the relative difference between the minimum (located at approximately $222 \%$ for $\alpha \simeq 550$ ) and the value of $\widetilde{\eta}_{\mathcal{T}}$ for $\alpha=0, \widetilde{\eta}_{\mathcal{T}} \simeq 224 \%$ is negligible. Thus, for this approximation space $\mathbb{H}_{h}$, it seems advantageous to use the Laplacian as the alternative operator.

Figure 5 shows the evolution of $\widetilde{\eta}_{\mathcal{T}}$ as a function of $\alpha$ under the same conditions as in Figure 3 but, in here, we modify the approximation space $\mathbb{H}_{h}$ by increasing the polynomial order $p$ by one. These 

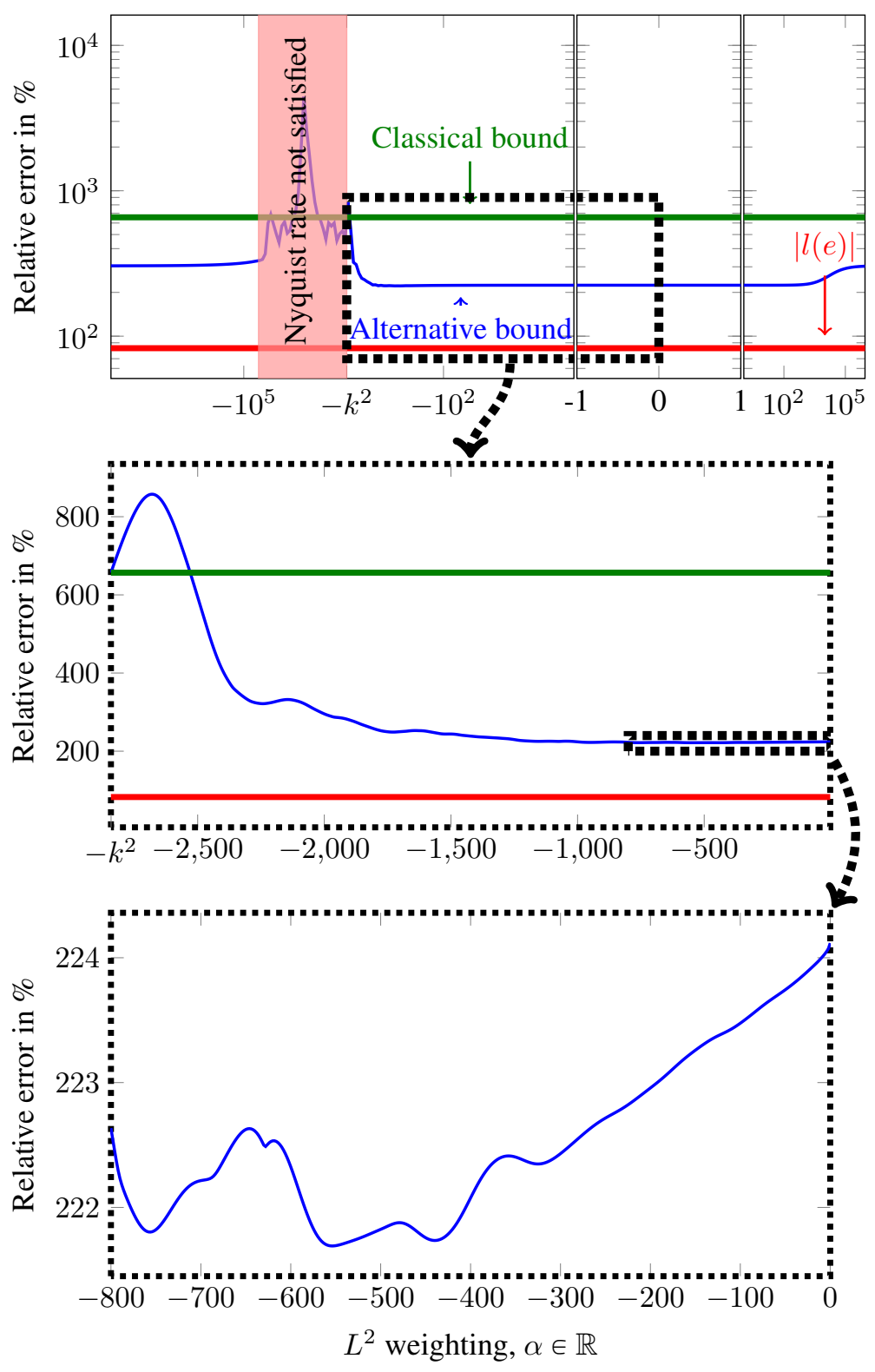

Figure 3: 2D case, $\widetilde{\eta}_{\mathcal{T}}$ versus $\alpha$ for $\alpha \in \mathbb{R}, k=2 \pi \times 8.5=17 \pi \simeq 53$, and around 3 DoF per wavelength (with uniform $p=3$ )

results show the robustness of the selected alternative operator (namely, the Laplacian) with respect to the choice of discrete space $\mathbb{H}_{h}$.

From those numerical results, it appears that the stability of the alternative operator plays a key role on the sharpness (or not) of the upper bounds. Indeed, unlike the Helmholtz operator, the Laplace one does not generate any dispersion error. Thus, in the remainder of this paper, we select the Laplacian as our alternative operator.

\section{4 p-Adaptive algorithm and implementation}

\section{$4.1 \quad p$-Adaptive algorithm}

We consider in this work a $p$-adaptive strategy in which the polynomial degree in the elements that are marked for refinement is increased by one. We start the iterative mesh-adaptation algorithm by defining an initial coarse mesh with a given mesh size $h$ and polynomial order $p$. The fine mesh is obtained by increasing uniformly the polynomial order of the coarse grid by $\Delta p=2$, as mentioned above. We compute the solutions of the direct (3.1) and the adjoint problems (3.2) on both meshes (coarse and fine). Then, 


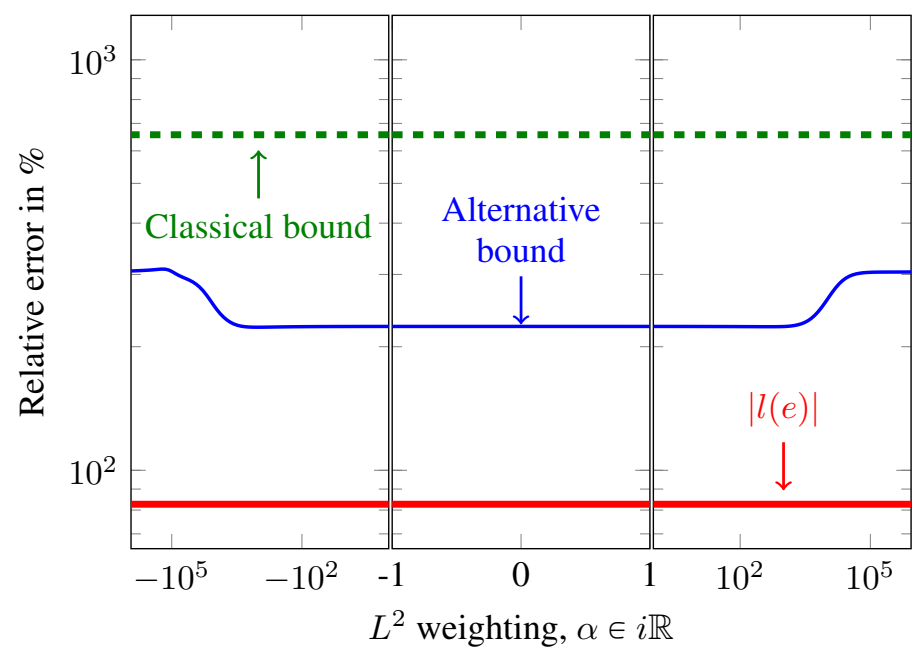

Figure 4: $2 \mathrm{D}$ case, $\widetilde{\eta}_{\mathcal{T}}(\alpha)$ for $\alpha \in i \mathbb{R}, k \simeq 17 \pi$, and around 3 DoF per wavelength (with uniform $p=3$ )

we approximate errors $e$ and $\varepsilon$ by computing the difference between the coarse and fine mesh solutions. We also estimate the alternative error $\widetilde{\varepsilon}$ by solving, on the fine grid, the alternative adjoint residual problem (3.6).

Once all error functions are estimated globally, we compute: (a) the element-wise contributions leading to the error representations (3.5) and (3.7), and (b) the local estimators used to perform adaptivity.

With above data, the adaptivity is performed as follows: we use the local error estimators $\eta_{K}$ or $\widetilde{\eta}_{K}$ to determine which elements need to be refined. We select all elements whose estimator is larger than a given percentage of the largest estimator $\max _{K}\left(\eta_{K}\right)$ and $\max _{K}\left(\widetilde{\eta}_{K}\right)$, respectively. In this work, we set that percentage to $40 \%$. We then isotropically increase by $\Delta p=1$ the polynomial orders of the selected elements, and we ensure the minimum rule [40]. This adaptive approach can be trivially implemented, and we use it here to illustrate the advantages and limitations of using alternative error representations. Of course, more sophisticated and effective adaptive processes can be used, e.g., [51, 52, 53, 54, 55].

The adaptive process gives us an updated coarse mesh, which constitutes the initial mesh for the subsequent adaptive iteration. We repeat this process until the required precision is reach. This algorithm is sketched in Figure 6.

\subsection{Implementation details}

The method has been implemented in Fortran90 using PETSc libraries [56] for the parallel resolution of the finite element systems. Since we need to compute the difference between the coarse and fine mesh solutions, we have to represent the coarse solution into the fine mesh data structure. Due to the use of hierarchical basis functions, the corresponding injection operator is trivially implemented by simply adding zeros to the coefficients associated with the $p+1$ and $p+2 \mathrm{DoF}$, leaving the remaining coefficients unchanged.

Following [40], we have implemented the 2D Projection Based Interpolation (PBI) in order to compare it with our method. The PBI ensures, for instance, almost optimal convergence rates (up to a logarithmic factor, see [57]) for the classical $p$-adaptive algorithm. Note that the quantities (3.5) and (3.7) computed using the PBI are no longer upper bounds of the error in the QoI. They are referred to as error indicators in the literature (cf. [58]). The PBI allows to project the fine mesh solution into the coarse grid by combining interpolation with a local minimum energy projection.

In order to compute the alternative error estimator, we need to build and factorize the matrix associated with the alternative operator. Thus, we cannot reuse the same matrix for the computation of both direct and alternative adjoint errors. This is a drawback of our method, hopefully compensated by designing an adaptive process that requires fewer DoF to reach its goal. Moreover, since the user selects the alternative operator, it is often the case that it can be solved with high precision via a fast (analytical or semi-analytical) method. 

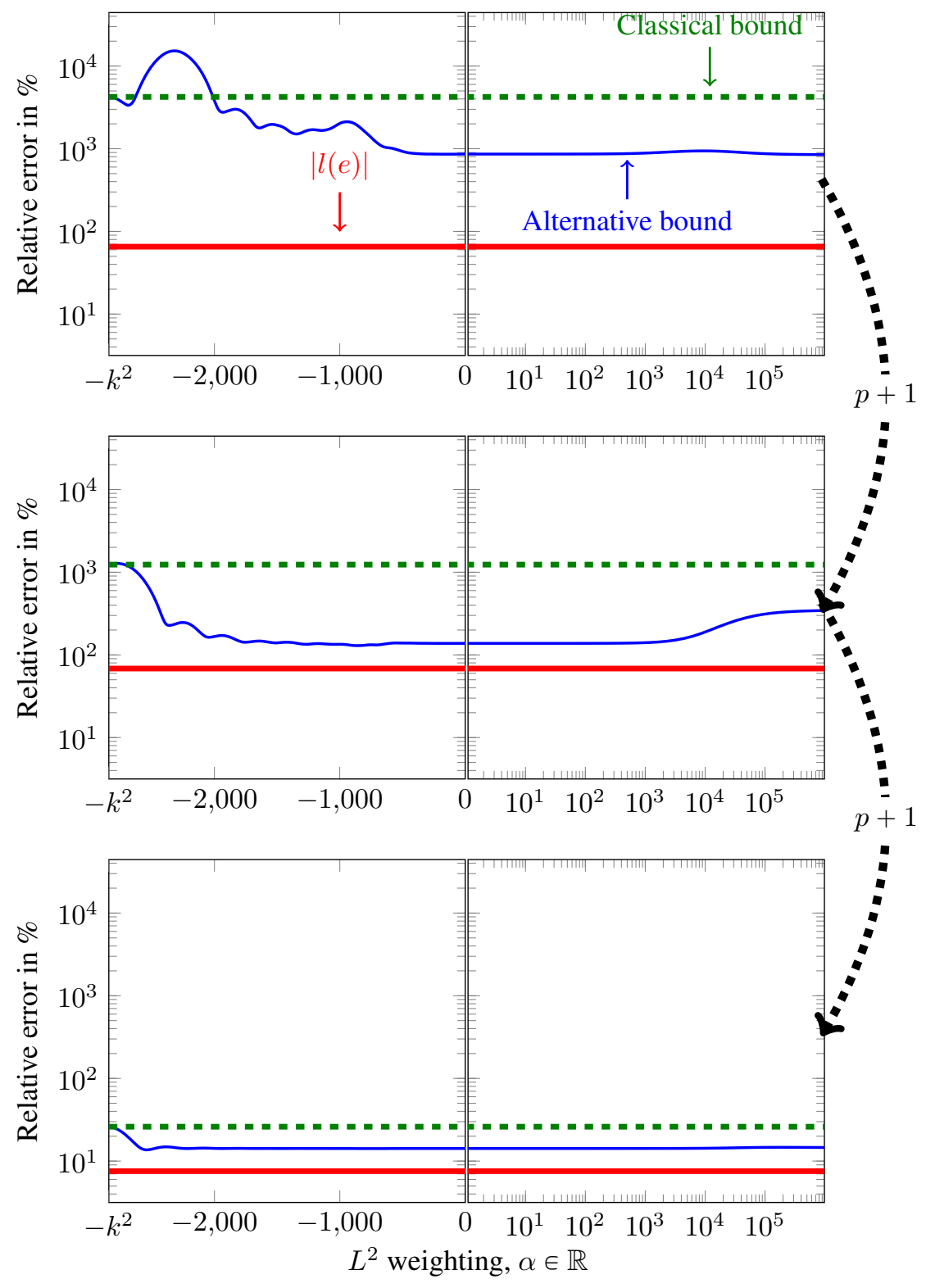

Figure 5: 2D case, $\widetilde{\eta}_{\mathcal{T}}$ versus $\alpha$ for $\alpha \in\left[-k^{2},+\infty[, k=17 \pi\right.$. The top graph is produced for 3 DoF per wavelength (with uniform $p=2$ ). The next graphs are obtained by increasing the approximation order $p$. The Laplace operator provides a sharper upper bound for all cases.

\section{$5 \quad$ Numerical results}

In this section, we present the results of numerical experiments in two and three spatial dimensions.

\subsection{D numerical results}

Figure 7 shows the upper bounds of the algorithm described in Figure 6 when performing uniform $h$-refinements (Figure 7a) and uniform $p$-refinements (Figure 7b). As mentioned in Section 3, Figure 7b shows that using $\widetilde{e}$ or $\widetilde{\varepsilon}$ leads to almost identical results. Thus, we restrict ourselves to the use of $\widetilde{\varepsilon}$, and the representation of the dual residual $\mathcal{R}_{d}^{h}$. In all cases, the size $h$ of the initial mesh elements is selected to enforce that the discretization exhibits always at least 2.5 DoF per wavelength $\left(p_{\text {init }}=1\right.$, uniformly). In this way, the Nyquist criterion is satisfied, and the error eventually decays at exponential rate with respect to $p$, since $2 p_{\text {init }}+1>k h+\beta(k h)^{1 / 3}$ for some $\beta>0$ (see $[59,58]$ ). Once the pollution error vanishes, the expected rate of convergence is $h^{2 p}$ (see [51]) for smooth enough solutions. Figure $7 \mathrm{~b}$ shows that the convergence rates obtained for this example when using uniform $p$-refinements are smaller than expected probably due to the loss of smoothness caused by the non smooth squared shape of the domain 


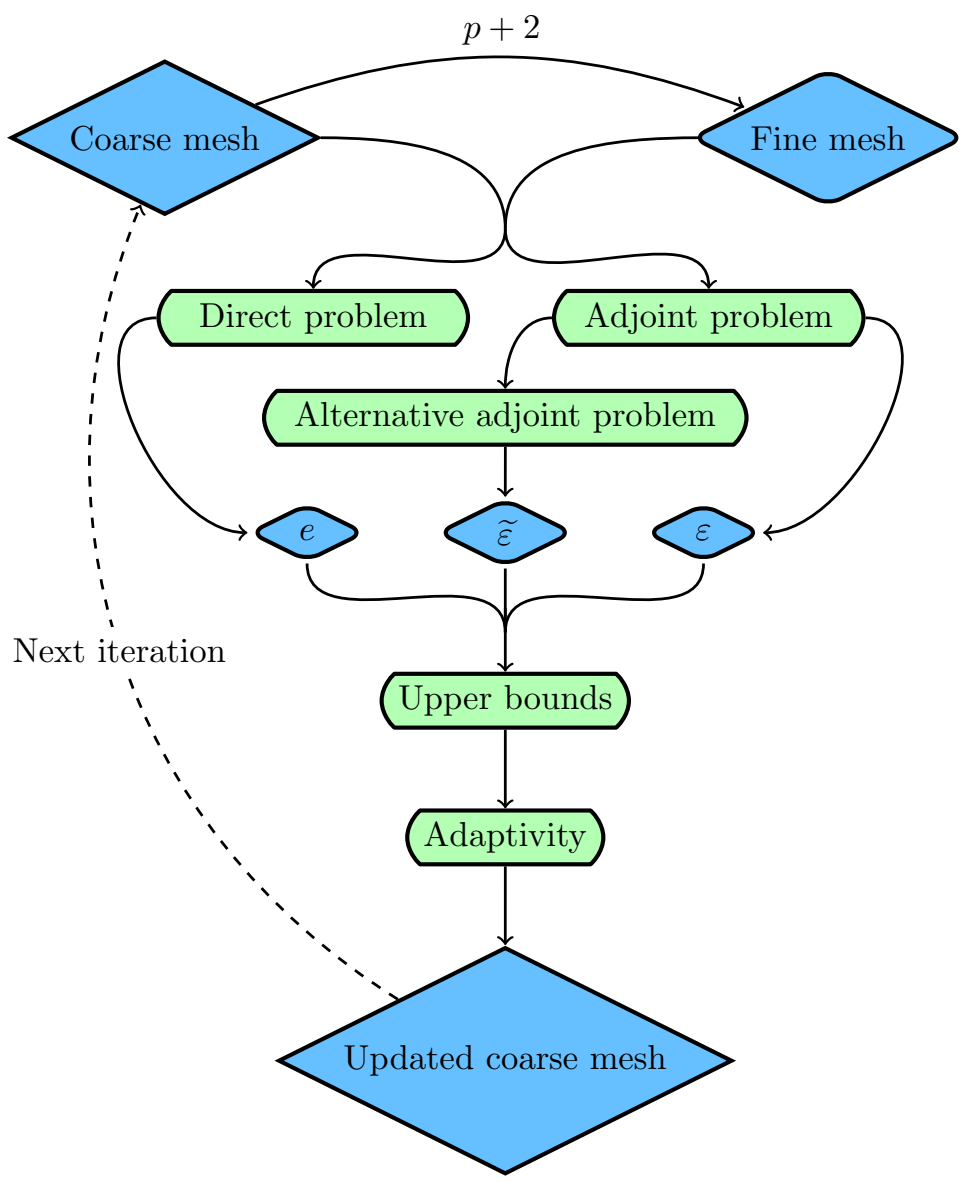

Figure 6: Algorithm of the GOA

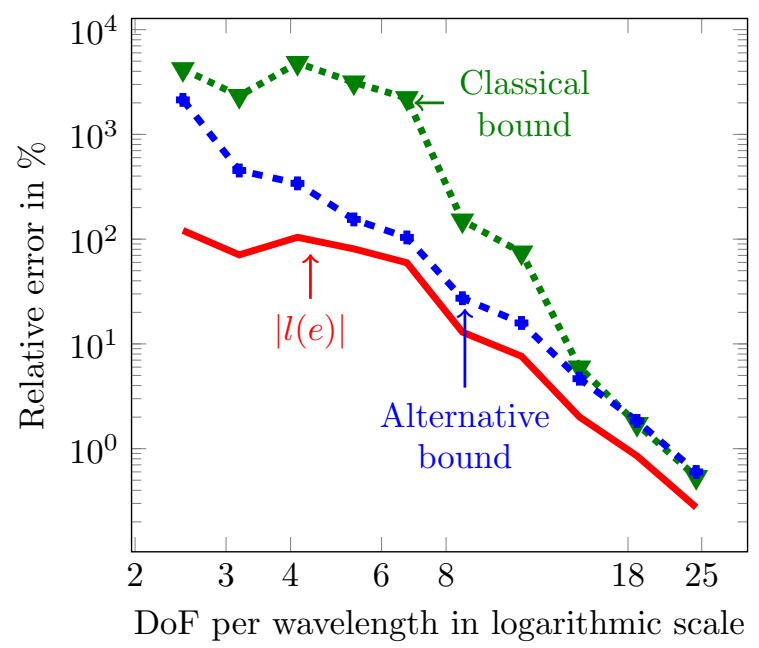

(a) Uniform $h$-refinements, $p=2$.

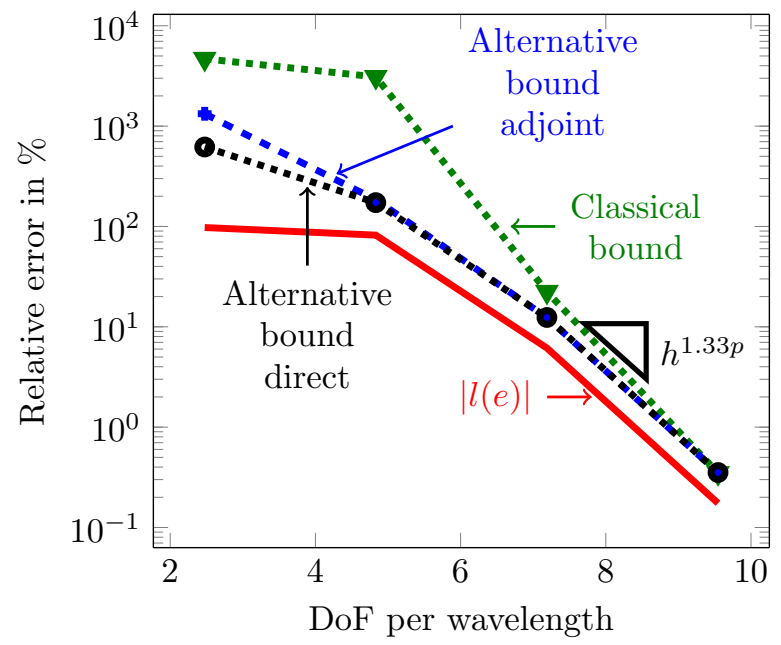

(b) Uniform $p$-refinements, $h k \gg 1$.

Figure 7: 2D case, $k=17 \pi$. Error evolution in the QoI — and the upper bounds given by the different error representations, namely, the classical bound (3.5) $\nabla^{-} \cdot$, the alternative bound using the residual dual $\mathcal{R}_{d}^{h}(3.7)=-\mathbf{*}$, and the alternative bound using the residual primal $\mathcal{R}_{p}^{h}(3.7)$

and the lack of regularity of the right-hand side.

From these graphics, we observe that the alternative upper bound (3.7) is much sharper in the preasymptotic range than the classical one (3.5), and both upper bounds coincide when the error in the QoI is around $1 \%$ or below (asymptotic regime). 
Figure 8 shows that with the alternative error representation, the $p$-adaptive algorithm converges without the need to introduce the PBI operator. When using the PBI, both algorithms converge with a similar behavior, as illustrated in Figure 8a. We emphasize that the naive adaptive process we considered causes the classical method to fail at converging. However, it converges when considering the alternative error representations.

When convergence occurs, errors exhibit almost identical convergence rates as those observed for the uniform $p$-refinements, see Figure $8 \mathrm{~b}$. Indeed, since the solution of our model is highly regular and of uniform amplitude, quasi-optimal meshes are obtained via uniform $p$-refinements, and the final adapted meshes (displayed in Figures 9b, 9c and 9d) are almost $p$-uniform. We remark that, with the classical criterion, the adaptive process does not converge because it selects inappropriate $p$-refinements that do not decrease the error, and the algorithm stops due to the limit of $p=14$ set on the approximation order; see Figure 9a.

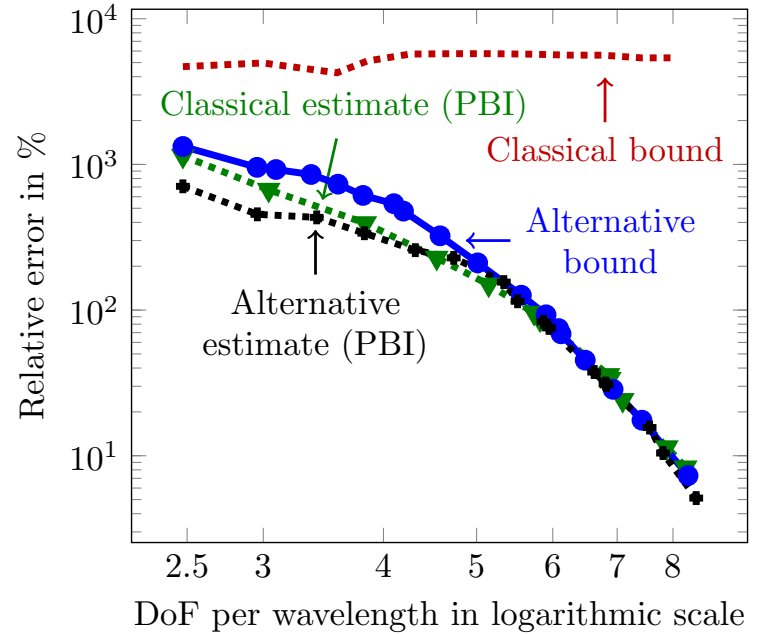

(a) Error representations.

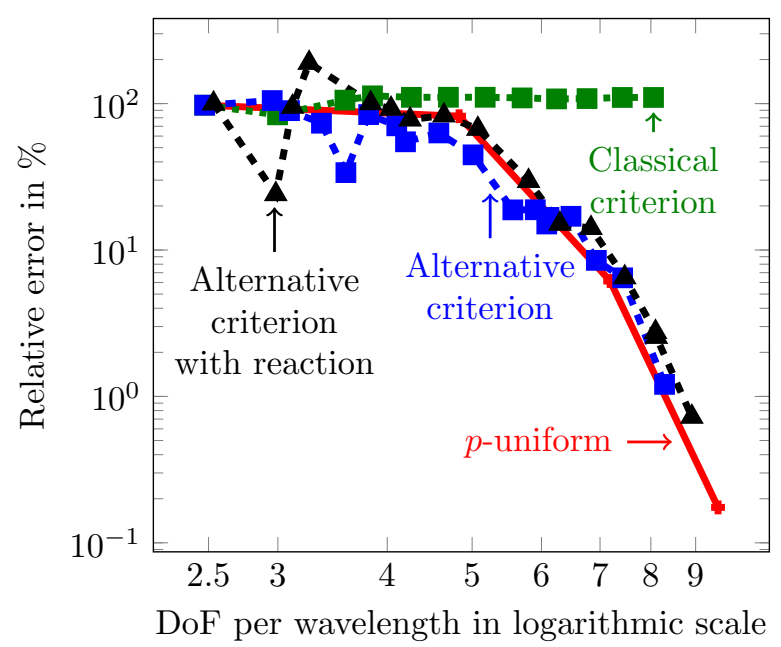

(b) Relative error (in \%) in the QoI: $100 \cdot|l(e)| /|l(u)|$.

Figure 8: 2D case, $k=17 \pi, h k \gg 1$. Panel (a): error representation for $p$-adaptivity depending on the criterion used: classical criterion $\cdot \cdot \cdot \cdot \cdot$, alternative criterion $\boldsymbol{-}-$, classical one using PBI $\cdot \boldsymbol{\nabla} \cdot$ ', and alternative one using PBI -**-. Panel (b): error evolution in the QoI, $|l(e)|$ depending on the adaptivity criterion: uniform $p$-refinements $\longrightarrow, p$-adaptivity using the classical error representation $\cdot$ - " $p$-adaptivity using the alternative error representation based on the Laplacian( - $\mathbf{0}$ ), and $p$-adaptivity using the alternative error representation based on the Laplace operator plus a positive reaction term with its coefficient being equal to $k^{2}(\boldsymbol{- A}-)$.

With the alternative error representation (where $\widetilde{B}$ is the weak Laplace operator) without PBI, we achieve similar results as those obtained with the classical estimate with PBI. Thus, we can substitute the PBI, which complicates the implementation, by the alternative dual problem.

Figure 10 illustrates the distribution map of the element error estimators $\eta_{K}(3.5)$ and $\widetilde{\eta_{K}}(3.7)$. We observe that the maximum error is about one order of magnitude larger for the classical estimators, which corroborates the results described in Figure 7. We additionally observe that the alternative method concentrates the largest errors close to the QoI, and they seem to rapidly decay as we move below $(0,1) \times\{0.75\}$. This observation is coherent with the fact that the error $\widetilde{\varepsilon}$ is the solution of a diffusive problem that takes as a source the residual of the adjoint problem. Conversely, the classical method exhibits large errors in a region that seems unrelated to the QoI. Figure 9a confirms that refinements occur in the aforementioned region. Apart from the extreme values, the error distribution is quite uniform, which is consistent with the nature of both $e$ and $\varepsilon$, which are solutions of Helmholtz residual problems.

\subsection{Increasing the wavenumber}

In this section, we increase the wavenumber $k$ of operator $B$. We employ a constant number of DoF per wavelength in order to compare the behavior of the classical and the alternative error upper bounds. Figure 11 illustrates that the alternative bound is significantly less affected by pollution than the classical one and stays closer to the error in the QoI. The observed oscillations are due to the selected QoI and depend upon the total number of wavelengths in the computational domain. 


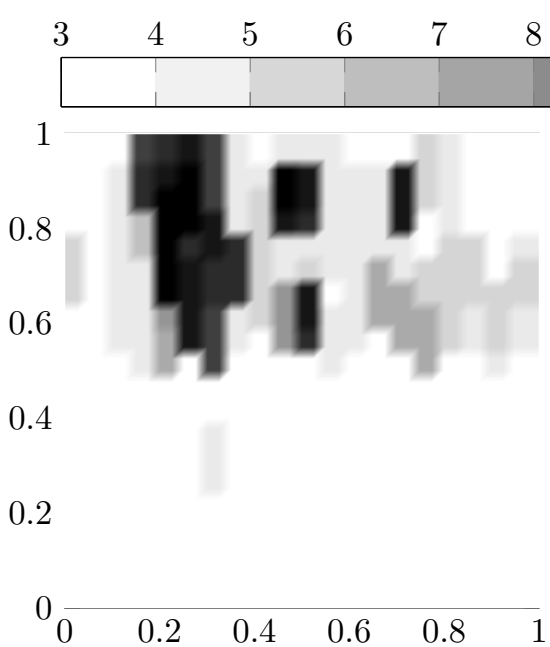

(a) Classical criterion without PBI

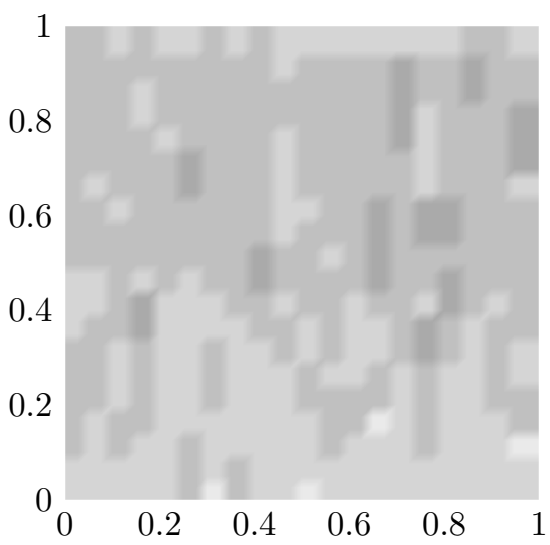

(c) Classical criterion with PBI

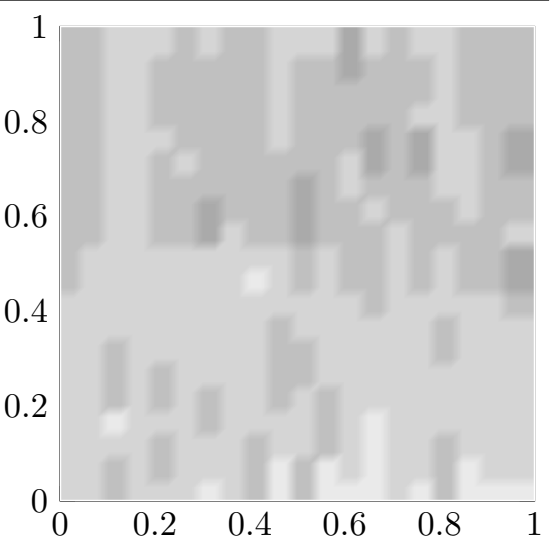

(b) Alternative criterion without PBI

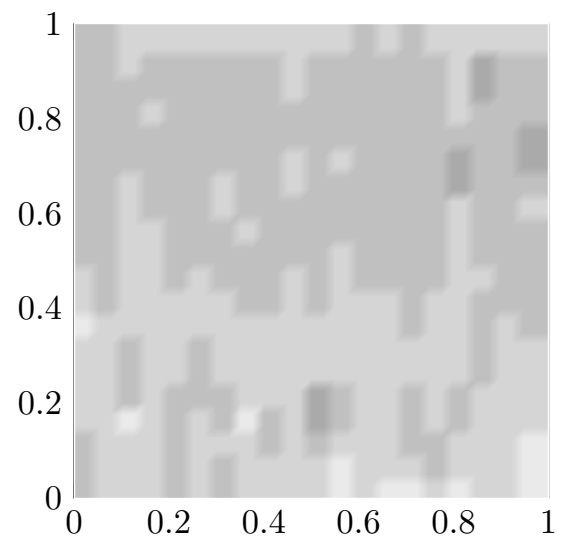

(d) Alternative criterion with PBI

Figure 9: 2D case, $k=17 \pi, h k \gg 1$. Final adapted fine meshes after $p$-adaptivity.

\subsection{Gradient of the solution as quantity of interest}

In this section, we show the robustness of the method by considering a different kind of QoI (similar to that used in [60]). For this purpose, we consider the 2D problem described in Section 2 with the following QoI:

$$
\langle l, w\rangle_{\mathbb{H}^{*}, \mathbb{H}}=\langle 1, \nabla w\rangle_{L^{2}\left(\Omega_{\mathrm{QoI}}\right)} \quad \forall w \in \mathbb{H} .
$$

where $\Omega_{\mathrm{QoI}}:=(0.75,1)^{2}$. Figure 12 shows that the upper bound provided by the alternative representation is sharper than the classical one in the pre-asymptotic regime, as expected.

\subsection{D numerical results}

Figure 13a shows numerical results corresponding to uniform $p$-refinements. Again, our method provides sharper upper bounds in the pre-asymptotic regime than those obtained with the classical method. The $p$-adaptive algorithm also exhibits a behavior similar to that observed in the 2D case (see Figure 13b). The alternative upper bound is driving the convergence more efficiently than the classical one.

\section{Conclusions}

This work generalizes the classical goal-oriented procedure described in [36, 34] by introducing an alternative operator for representing the error.

We extend the results of our previous publication [38] to the multi-dimensional Helmholtz problem. We address the question of whether we can find an operator that provides the sharpest upper bounds 


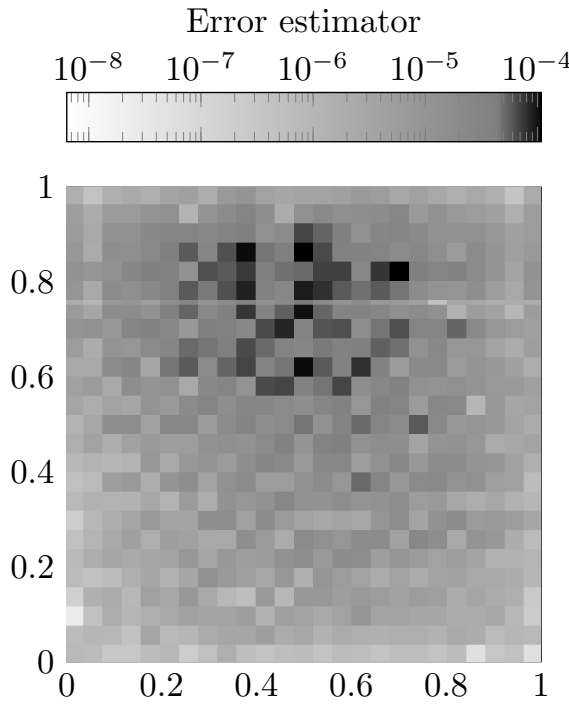

(a) Classical estimators

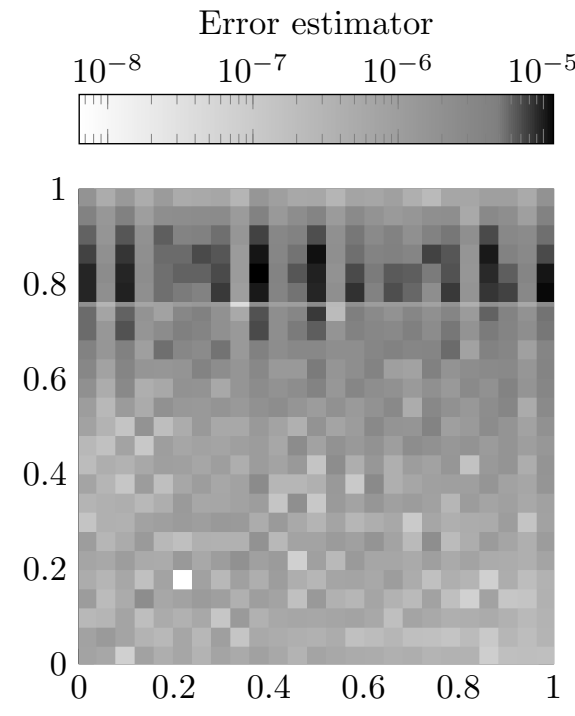

(b) Alternative estimators

Figure 10: 2D case, $k=17 \pi, p=1$ uniformly, $h k \gg 1$. Error map representations. We select a logarithmic scale of the error for selecting the color. The gray color has been set to separate between the elements that are to be refined (those with darker tones) and those that will be unrefined (brighter tones).

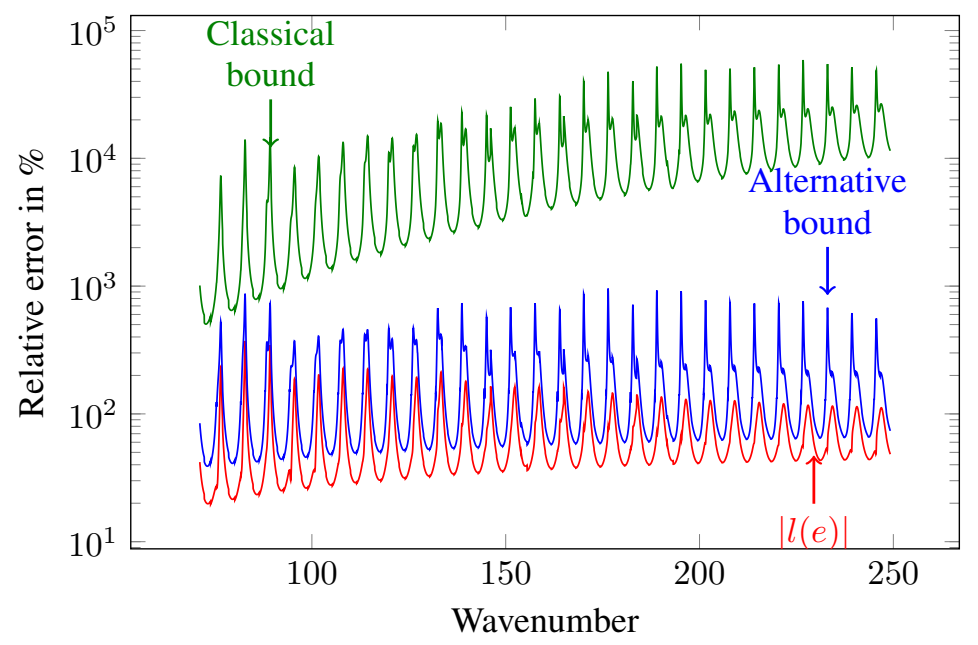

Figure 11: 2D case, uniform $p=2 ; h$ is determined in order to ensure a fixed number of (approx. eight) DoF per wavelength. Error evolution in the QoI and upper bounds for the error representation of the QoI when the wavenumber is increasing from 71 to 250 . $B$ is the Laplace operator.

independently of the approximation space. Since this seems to be prohibitively expensive, we propose to use the Laplacian as the alternative operator in order to represent the dual residual. With that operator, we are not computing the sharpest upper bound, but it provides a good trade-off between computational feasibility and obtaining sharp upper bounds.

As mentioned in Section 3, a guideline for the choice of the alternative operator is to construct an operator that presents a better numerical stability than the original one.

Numerical results show that error upper bounds are sharper when using the alternative representation than with the classical one. As a result, our simple adaptive process does not need the PBI (nor any other projection) to converge when using the alternative error representation as indicators, whereas the classical representation fails to drive the algorithm to converge without the PBI. The classical and alternative upper bounds coincide when reaching the asymptotic regime. As an extension of this work, we applied the developed method to (a) a convection-dominated problem (see Appendix A), (b) problems with discontinuous material coefficients (see Appendix B), and (c) a geophysical application consisting of simulating sonic logging-while-drilling (LWD) measurements (see Appendix C). We draw the same 


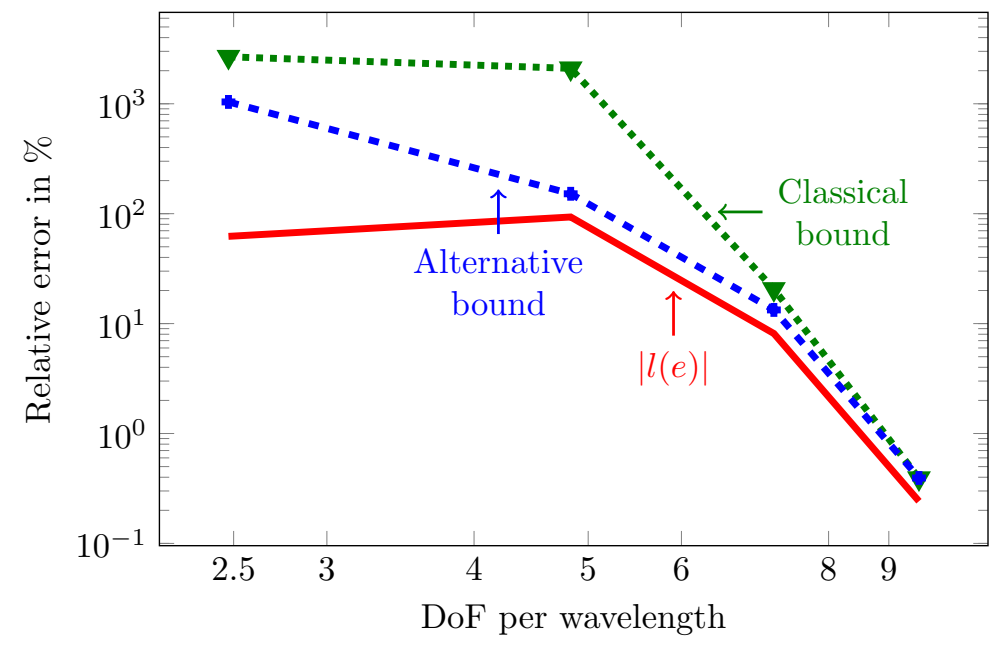

Figure 12: 2D case, $k=17 \pi, h k \gg 1$, uniform $p$-refinements. Error evolution in the QoI and upper bounds for the error representation of the QoI when the QoI is the average of the gradient on a subdomain of $\Omega$.

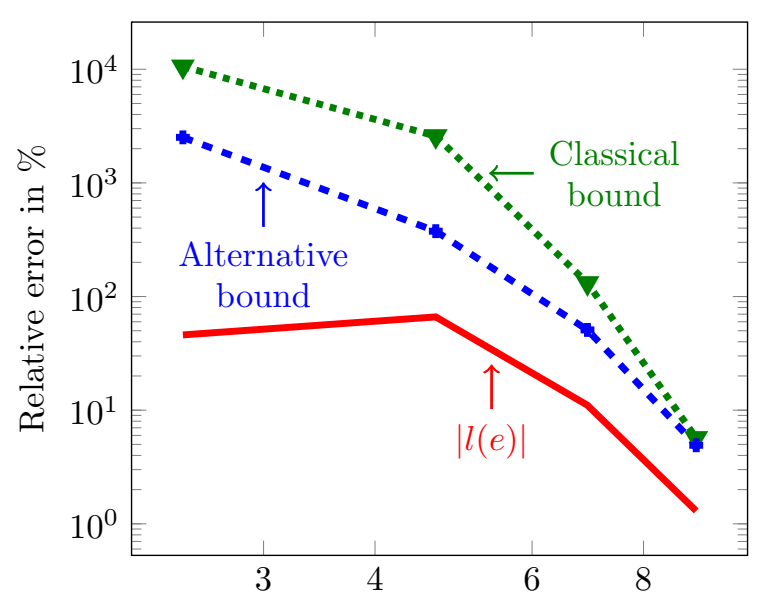

DoF per wavelength in logarithmic scale

(a) Uniform $p$-refinements.

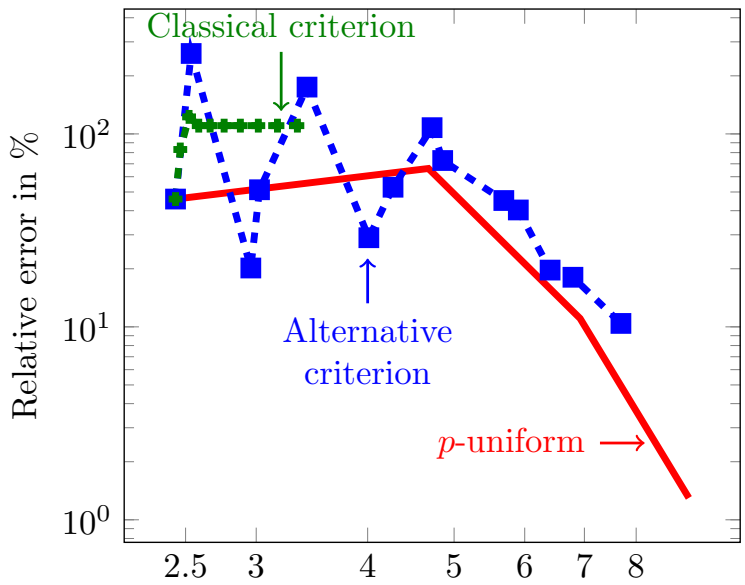

DoF per wavelength in logarithmic scale

(b) Error evolution in the QoI depending on the $p$ adaptive criterion.

Figure 13: 3D case, $k=6 \sqrt{3} \pi \simeq 32.64$.

conclusions in all these examples.

One notorious advantage of our approach is its flexibility. Indeed, we can apply this technique to a wide range of problems, including adaptivity in time domain [61,62] or $h p$-adaptive algorithms $[4,6]$. We are also working on extending the proposed approach to other discretizations such as Petrov-Galerkin, Discontinuous Galerkin, and/or some version of the Discontinuous Petrov Galerkin method.

\section{A Convection dominated diffusion problem}

We consider the following model problem based on a convection dominated diffusion equation. For $\Omega=(0,1)^{2}$,

Find $u$ such that, for $\nu>0$,

$$
\begin{cases}-\nu \Delta u+(1,1) \cdot \nabla u=1 & \text { on } \Omega, \\ u=0 & \text { on } \partial \Omega .\end{cases}
$$

We set $\mathbb{H}=\left\{u \in H^{1}(\Omega), u=0\right.$ on $\left.\partial \Omega\right\}$ and $\langle\cdot, \cdot\rangle_{L^{2}}$ the standard $L^{2}$ scalar product. We define the QoI 
as the integral on $\Omega_{\mathrm{QoI}} \subset \Omega$, which is given by the functional:

$$
\langle l, w\rangle_{\mathbb{H}^{*}, \mathbb{H}}=\langle 1, w\rangle_{L^{2}\left(\Omega_{\mathrm{QoI}}\right)} \quad \forall w \in \mathbb{H} .
$$

Operator $B \in \mathcal{L}\left(\mathbb{H}, \mathbb{H}^{*}\right)$ is defined as follows,

$$
\langle B w, z\rangle_{\mathbb{H}^{*}, \mathbb{H}}=\nu\langle\nabla w, \nabla z\rangle_{L^{2}(\Omega)}+\langle(1,1) \cdot \nabla w, z\rangle_{L^{2}(\Omega)}, \quad \forall w, z \in \mathbb{H} .
$$

We define the alternative operator:

$$
\langle\widetilde{B} w, z\rangle_{\mathbb{H}^{*}, \mathbb{H}}=\langle\nabla w, \nabla z\rangle_{L^{2}(\Omega)} \quad \forall w, z \in \mathbb{H},
$$

which is the one associated with the Laplace equation with homogeneous Dirichlet boundary conditions on $\partial \Omega$. We set the QoI domain to $\Omega_{\mathrm{QoI}}=(0.75,1)^{2}$, so part of the boundary layer is included. We perform uniform $p$ - and $h$-refinements starting from a quasi-uniform initial mesh with $11 \times 11$ elements.

Figure 14 shows the behavior of the upper bounds with respect to diffusion parameter $\nu$ : as $\nu$ decreases, the bounds increase, as expected.

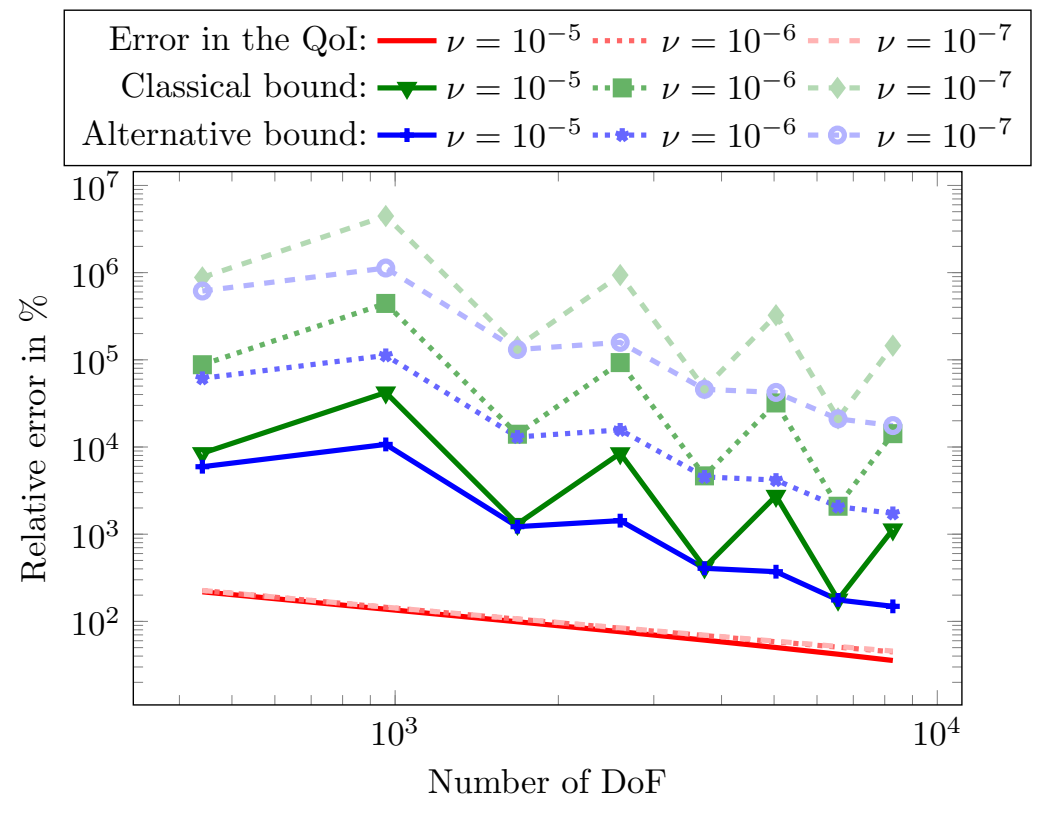

Figure 14: 2D case, convection-dominated diffusion problem. Uniform $p$-refinements varying the diffusion coefficient.

We now set $\nu=10^{-4}$ and consider uniform $p$-refinements. Figure 15a shows the following four upper bounds of the error in the QoI:

$$
\begin{aligned}
|l(e)| & \leqslant \sum_{K}\left|\left\langle B_{K} e, \varepsilon\right\rangle\right| \\
& \leqslant \sum_{K}\left(\nu\|\nabla \varepsilon\|_{L^{2}(K)}+\|\varepsilon\|_{L^{2}(K)}\right)\|\nabla e\|_{L^{2}(K)}
\end{aligned}
$$

and

$$
\begin{aligned}
|l(e)| & \leqslant \sum_{K}\left|\left\langle\widetilde{B}_{K} e, \widetilde{\varepsilon}\right\rangle\right| \\
& \leqslant \sum_{K}\|\nabla e\|_{L^{2}(K)}\|\nabla \widetilde{\varepsilon}\|_{L^{2}(K)}
\end{aligned}
$$

Upper bounds (A.1) and (A.3) are non monotonous probably due to the lack of control on the angle between the errors. However, the monotonicity is recovered when considering the upper bounds (A.2) and (A.4), although those upper bounds are less sharp. In all cases, we nevertheless observe that the alternative upper bounds are sharper than the classical ones. Moreover, if $\widetilde{B}$ also includes an $L^{2}$ inner product term as follows,

$$
\langle\widetilde{B} w, z\rangle_{\mathbb{H}^{*}, \mathbb{H}}=\langle\nabla w, \nabla z\rangle_{L^{2}(\Omega)}+\langle w, z\rangle_{L^{2}(\Omega)} \quad \forall w, z \in \mathbb{H},
$$




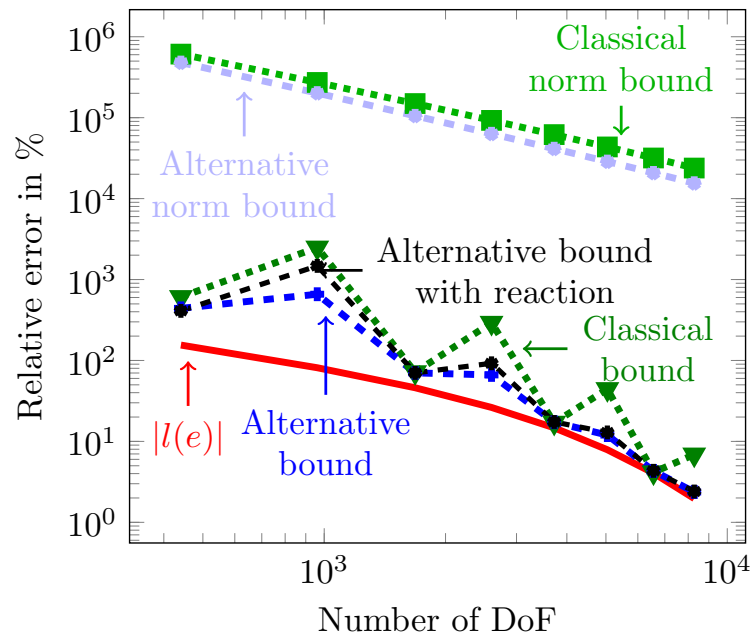

(a) Uniform $p$-refinement.

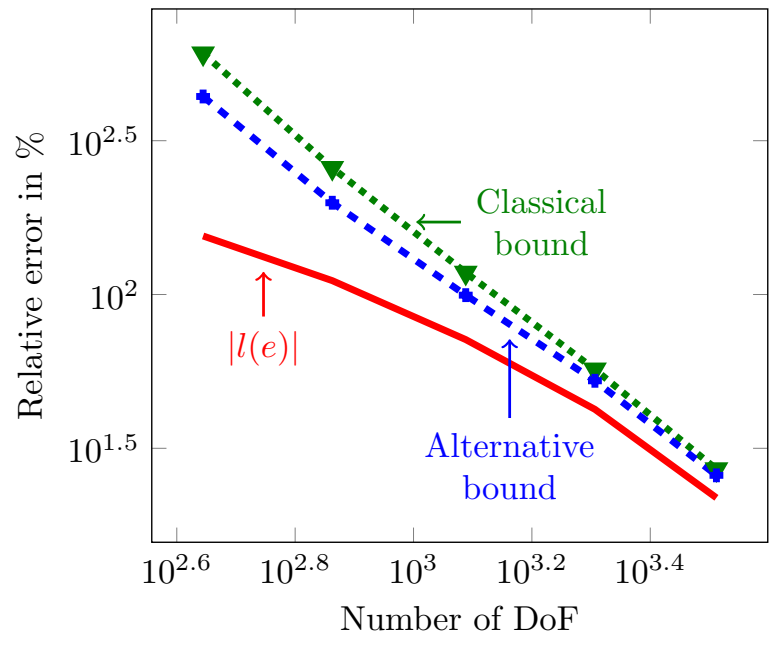

(b) Uniform $h$-refinements.

Figure 15: 2D case, convection-dominated diffusion problem. Uniform refinements for $\nu=10^{-4}$.

the alternative bound, it is still sharper than the classical one. Nonetheless, the Laplace alternative operator still provides the best results (see Figure 15a). For $h$-refinements (see Figure 15b), we observe that upper bound (A.3), although not sharp, is still sharper than upper bound (A.1). However, there is no oscillating behavior due to the selected mesh size. For a finer size, we again observe the previously mentioned oscillations.

We now execute the $p$-adaptive process for $\nu=10^{-7}$ with an initial mesh geometrically graded from the boundaries towards the interior of the domain in a way that the boundary layers are captured. Figure 16 shows the evolution of the upper bounds driving the adaptivity. The classical method does not converge, whereas the alternative criterion is successful even if not sharp at first glance. Additionally, the number of DoF needed for achieving a given precision is lower for the adaptive discretization than for the uniform refinement case. This occurs because features of the solution are localized unlike the solution of the Helmholtz problem

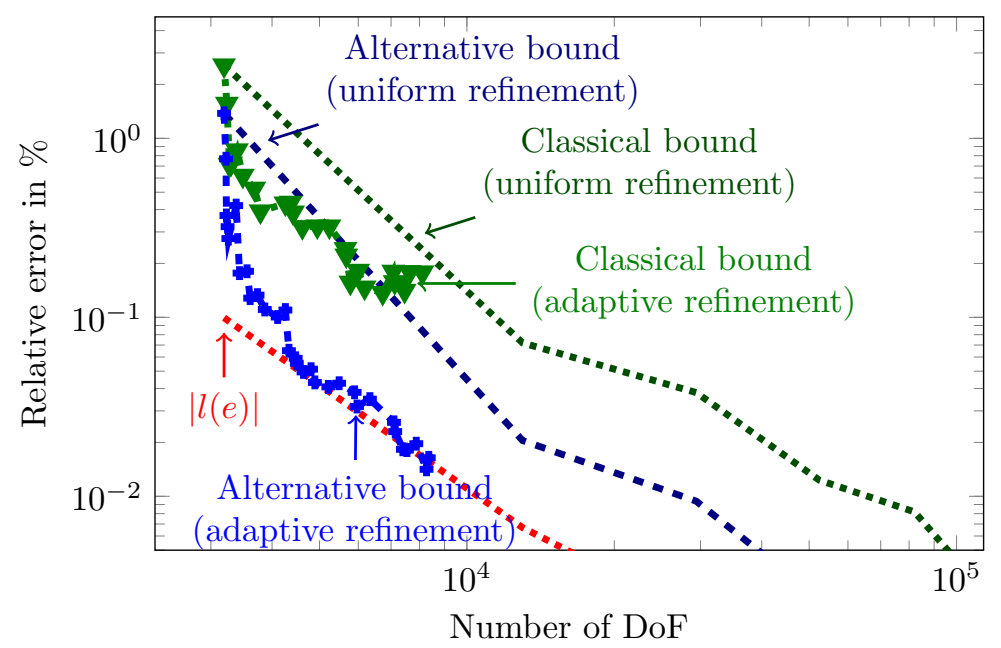

Figure 16: 2D case, convection-dominated diffusion. $p$-adaptivity with $\nu=10^{-7}$.

\section{B Discontinuous coefficients}

We consider the following general model problem governed by a diffusion convection reaction equation with mixed boundary conditions: For $\Omega=(0,1)^{2}$, with $\bar{\Gamma}_{D} \bigcup \bar{\Gamma}_{I}=\partial \Omega, \stackrel{\circ}{\Gamma}_{D} \bigcap \stackrel{\circ}{\Gamma}_{I}=\emptyset$. 
Find $u$ such that, for $\alpha, \beta, \gamma \in L^{2}(\Omega)$,

$$
\begin{cases}-\nabla(\alpha \cdot \nabla u)+\beta(1,1) \cdot \nabla u+\gamma u=1 & \text { in } \Omega, \\ u=0 & \text { on } \Gamma_{D}, \\ \partial_{n} u+i \sqrt{|\gamma|} u=0 & \text { on } \Gamma_{I},\end{cases}
$$

In particular, for $\Gamma_{I}=\emptyset$ and $\gamma=0$, we recover our previous convection diffusion problem, and for $\beta=0$ and $\gamma<0$, we have again the Helmholtz equation.

We set $\mathbb{H}:=\left\{u \in H^{1}(\Omega), u_{\mid \Gamma_{D}}=0\right\}$ and $\langle\cdot, \cdot\rangle_{L^{2}}$ the standard $L^{2}$ scalar product. We define the QoI as the integral on $\Omega_{\mathrm{QoI}} \subset \Omega$, which is given by the functional

$$
\langle l, w\rangle_{\mathbb{H}^{*}, \mathbb{H}}=\langle 1, w\rangle_{L^{2}\left(\Omega_{\mathrm{QoI}}\right)} \quad \forall w \in \mathbb{H} .
$$

In the following, we set the QoI domain to $\Omega_{\mathrm{QoI}}=(0.75,1)^{2}$. Operator $B \in \mathcal{L}\left(\mathbb{H}, \mathbb{H}^{*}\right)$ is defined as follows,

$$
\begin{aligned}
\langle B w, z\rangle_{\mathbb{H}^{*}, \mathbb{H}}= & \langle\alpha \nabla w, \nabla z\rangle_{L^{2}(\Omega)}+\langle\beta(1,1) \cdot \nabla w, z\rangle_{L^{2}(\Omega)} \\
& +\langle\gamma w, z\rangle_{L^{2}(\Omega)}+i\langle\sqrt{|\gamma|} w, z\rangle_{L^{2}\left(\Gamma_{I}\right)}, \quad \forall w, z \in \mathbb{H} .
\end{aligned}
$$

We define the following alternative operator: for $\widetilde{\alpha}, \widetilde{\beta}, \widetilde{\gamma} \in L^{2}(\Omega)$,

$$
\begin{aligned}
\langle\widetilde{B} w, z\rangle_{\mathbb{H}^{*}, \mathbb{H}}= & \langle\widetilde{\alpha} \nabla w, \nabla z\rangle_{L^{2}(\Omega)}+\langle\widetilde{\beta}(1,1) \cdot \nabla w, z\rangle_{L^{2}(\Omega)} \\
& +\langle\widetilde{\gamma} w, z\rangle_{L^{2}(\Omega)}+i\langle\sqrt{|\widetilde{\gamma}|} w, z\rangle_{L^{2}\left(\Gamma_{I}\right)}, \quad \forall w, z \in \mathbb{H} .
\end{aligned}
$$

The coefficients are considered to be piecewise-constant, as illustrated in Figure 17.

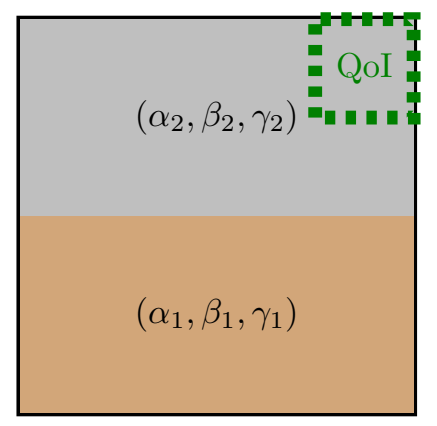

Figure 17: Domain with two materials.

We first set $\Gamma_{I}=\emptyset, \gamma=0, \beta=1$ with the following piecewise-constant diffusion coefficient: $\alpha=$ $\alpha_{1} \mathbb{1}_{(0,1) \times(0,0.5)}+\alpha_{2} \mathbb{1}_{(0,1) \times(0.5,1)}$. Figure 18a shows the upper bounds for $\alpha_{1}=10^{-4}, \alpha_{2}=10^{-7}, \widetilde{\beta}=\widetilde{\gamma}=0$, and $\widetilde{\alpha}=\alpha$. The alternative upper bound is sharper than the classical one. As the discontinuity in the coefficients induce a loss of stability, both upper bounds are less effective than in the constant coefficient case. However, the alternative upper bound is less affected than the classical one.

We now consider a Helmholtz problem by setting

$$
\Gamma_{I}=(\{1\} \times(0,1)) \bigcup((0,1) \times\{1\}),
$$

$\alpha=1, \beta=0$ and a piecewise constant reaction coefficient:

$$
\gamma=\gamma_{1} \mathbb{1}_{(0,1) \times(0,0.5)}+\gamma_{2} \mathbb{1}_{(0,1) \times(0.5,1)} .
$$

Figure 18b shows the upper bounds for $\gamma_{1}=-2842$ and $\gamma_{2}=-5053, \widetilde{\beta}=\widetilde{\gamma}=0$, and $\widetilde{\alpha}=\alpha$. For this case, there are no significant differences between continuous or discontinuous coefficients. The alternative method is producing sharper upper bounds for both cases, and we again observe that both bounds coincide in the asymptotic regime (when the error in the QoI is around 1\%). 


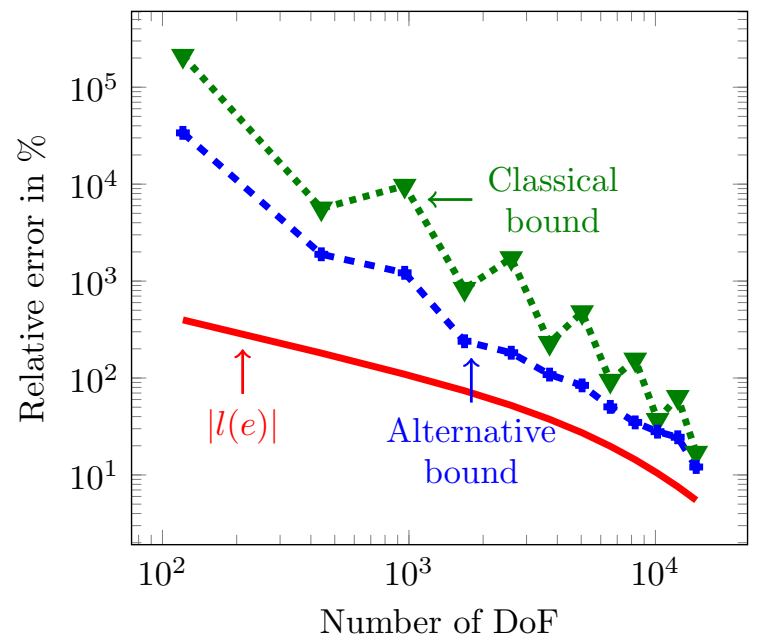

(a) Convection-dominated diffusion problem with a discontinuous diffusion coefficient $\left(\alpha=10^{-4}\right.$. $\left.\mathbb{1}_{(0,1) \times(0,0.5)}+10^{-7} \cdot \mathbb{1}_{(0,1) \times(0.5,1)}\right)$.

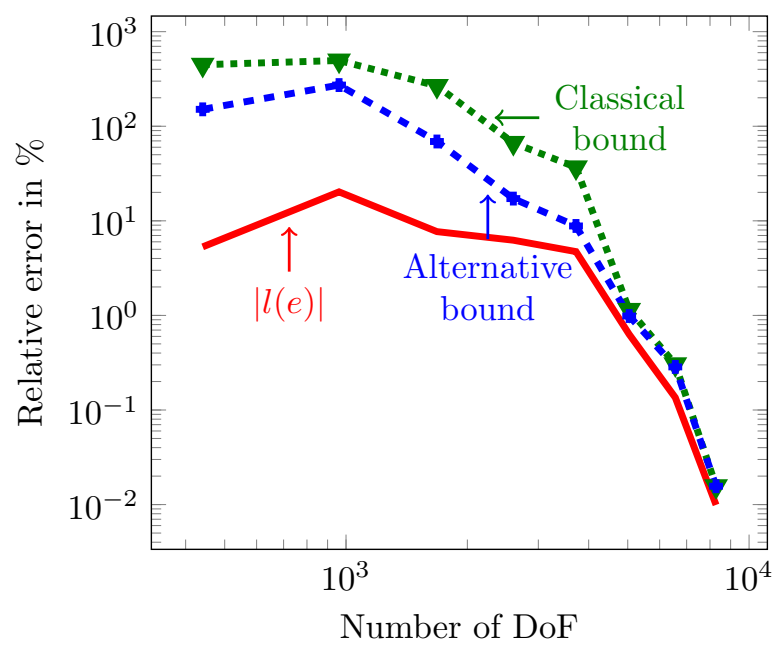

(b) Helmholtz problem with discontinuous materials $\left(\gamma=2842 \cdot \mathbb{1}_{(0,1) \times(0,0.5)}+5053 \cdot \mathbb{1}_{(0,1) \times(0.5,1)}\right)$.

Figure 18: 2D case. Upper bounds corresponding to the case of uniform $p$-refinements for discontinuous coefficients.

\section{Geophysical borehole application: frequency domain acous- tics}

In this appendix, we apply our adaptive strategy to the simulation of sonic logging-while-drilling (LWD) measurements, as described in $[63,64,65]$. To simplify the implementation, we focus only on a purely acoustic media (without elasticity).

\section{C.1 Model problem}

We assume axial symmetry around the center of the borehole, so we can reduce the original 3D formulation to two spatial dimensions using cylindrical coordinates. To truncate the computational domain, we employ a Perfectly Matched Layer (PML) (see [66]). The logging instrument contains a transmitter $t_{x}$ and an array of 13 receivers $\left(r_{x}^{i}\right)_{i=1,13}$.

We consider the following problem with mixed boundary conditions: for $\Omega \subset \mathbb{R}^{2}$, with boundary $\bar{\Gamma}_{D} \bigcup \bar{\Gamma}_{\text {axis }}=\partial \Omega, \stackrel{\circ}{\Gamma}_{D} \bigcap \stackrel{\circ}{\Gamma}_{\text {axis }}=\emptyset$, where $\Gamma_{\text {axis }}$ is the boundary corresponding to the symmetry axis.

$$
\begin{aligned}
& \text { Find } \mathfrak{p} \text { such that, for } c \in L^{\infty}(\Omega), c \neq 0, f \in \mathbb{R}^{+}, \\
& \qquad \begin{cases}-\Delta \mathfrak{p}-\frac{2 \pi \cdot f}{c} \mathfrak{p}=\mathbb{1}_{t_{x}} & \text { in } \Omega \\
\mathfrak{p}=0 & \text { on } \Gamma_{D}, \\
\nabla \mathfrak{p} \cdot \vec{n}=0 & \text { on } \Gamma_{\text {axis }},\end{cases}
\end{aligned}
$$

where $\mathbb{1}_{t_{x}}$ is the characteristic function over the area occupied by transmitter $t_{x}$ and $\vec{n}$ is the outgoing normal unit vector. We define the QoI as the sum over the array of receivers of the average value of the solution at each receiver.

$$
\langle l, \phi\rangle_{\mathbb{H}^{*}, \mathbb{H}}=\sum_{i=1}^{N_{r_{x}}} \frac{1}{\left|\Omega_{r_{x}^{i}}\right|} \int_{\Omega_{r_{x}^{i}}} \phi, \quad \forall \phi \in \mathbb{H}
$$

where $\Omega_{r_{x}^{i}}$ is the domain occupied by the $i$-th receiver. We set the frequency of the transmitter to $f=20$ $\mathrm{kHz}$. The coefficient $c$ varies throughout the domain depending on the propagation velocity of the wave in each layer. The domain is composed of three different layers: The tool (with associated velocity $c_{\text {tool }}$ ), the fluid surrounding the tool with velocity $c_{\text {fluid }}$, and the rock formation with velocity $c_{\text {formation }}$ (see Table 1). The computational domain is described in Figure 19. 


\begin{tabular}{|c|c|c|c|}
\hline & $c_{\text {tool }}$ & $c_{\text {fluid }}$ & $c_{\text {formation }}$ \\
\hline Velocities $(\mathrm{m} / \mathrm{s})$ & 5862 & 1524 & 4354 \\
\hline
\end{tabular}

Table 1: Propagation velocities $(\mathrm{m} / \mathrm{s})$ of the materials for LWD acoustic measurements.

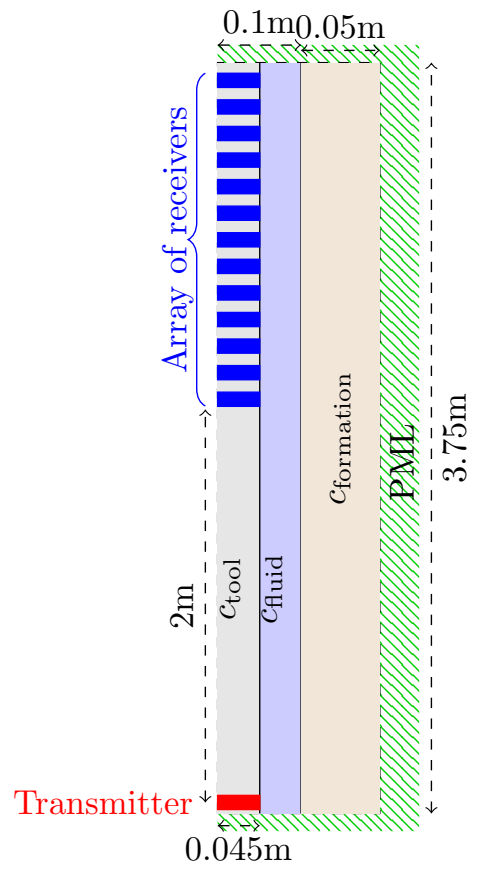

Figure 19: Sketch of the computational domain for acoustic LWD measurements, composed of three different materials: The logging tool, the borehole fluid, and the rock formation. The axis of symmetry is located on the left side of the domain, and coincides with the tool center. We have added a PML to truncate the computational domain. In red, we draw the transmitter and in blue the array of receivers.

\section{C.2 Numerical Results}

Figure 20 shows the evolution of the error bounds throughout the adaptive process. We plot upper bounds given by Eqs. (3.5) and (3.7) both when using $p$-adaptivity with and without the PBI operator (in the case of the PBI, the displayed curves are estimates rather than strict upper bounds). The results are similar to the ones of Figure 8a. The classical criterion fails to drive the adaptive process. On the other hand, both the alternative and the PBI criteria succeed. The resulting meshes are shown in Figure 21. We observe that the classical criterion (see Figure 21a) performs refinements only within the borehole, with special emphasis on the area occupied by the fluid. When the adaptive process is successful (see Figures 21b, 21c and 21d), refinements occur almost uniformly throughout the entire computational domain, except on the surroundings of the junctions between the PML, the logging instrument, and the fluid. The solution at those points is probably singular due to the large variation of the coefficients, and further refinements are required.

\section{References}

[1] Babuška I, Rheinboldt WC. A-posteriori error estimates for the finite element method. International Journal for Numerical Methods in Engineering 1978; 12(10):1597-1615.

[2] Babuška I, Rheinboldt W. Adaptive approaches and reliability estimations in finite element analysis. Computer Methods in Applied Mechanics and Engineering 1979; 17:519 - 540, doi:http://dx. doi.org/10.1016/0045-7825(79)90042-2. URL http://www.sciencedirect.com/science/article/ pii/0045782579900422.

[3] Pardo D. Integration of $h p$-adaptivity with a two grid solver: applications to electromagnetics. $\mathrm{PhD}$ Thesis, The University of Texas at Austin 2004. 


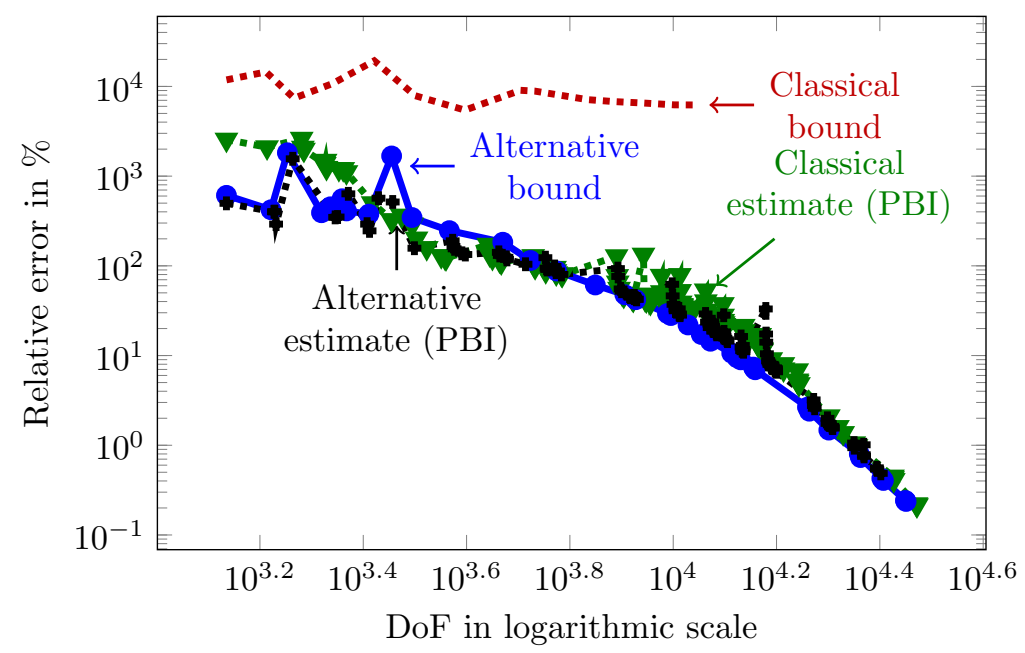

Figure 20: Sonic LWD: Error representations for p-adaptivity depending on the criterion used: classical criterion without PBI $\cdot . \cdot \cdot$, alternative criterion without PBI $\multimap-$, classical criterion using the PBI $\cdot \boldsymbol{\nabla} \cdot$ ', and alternative criterion using the PBI $\cdot \cdot * \cdot \cdot$.

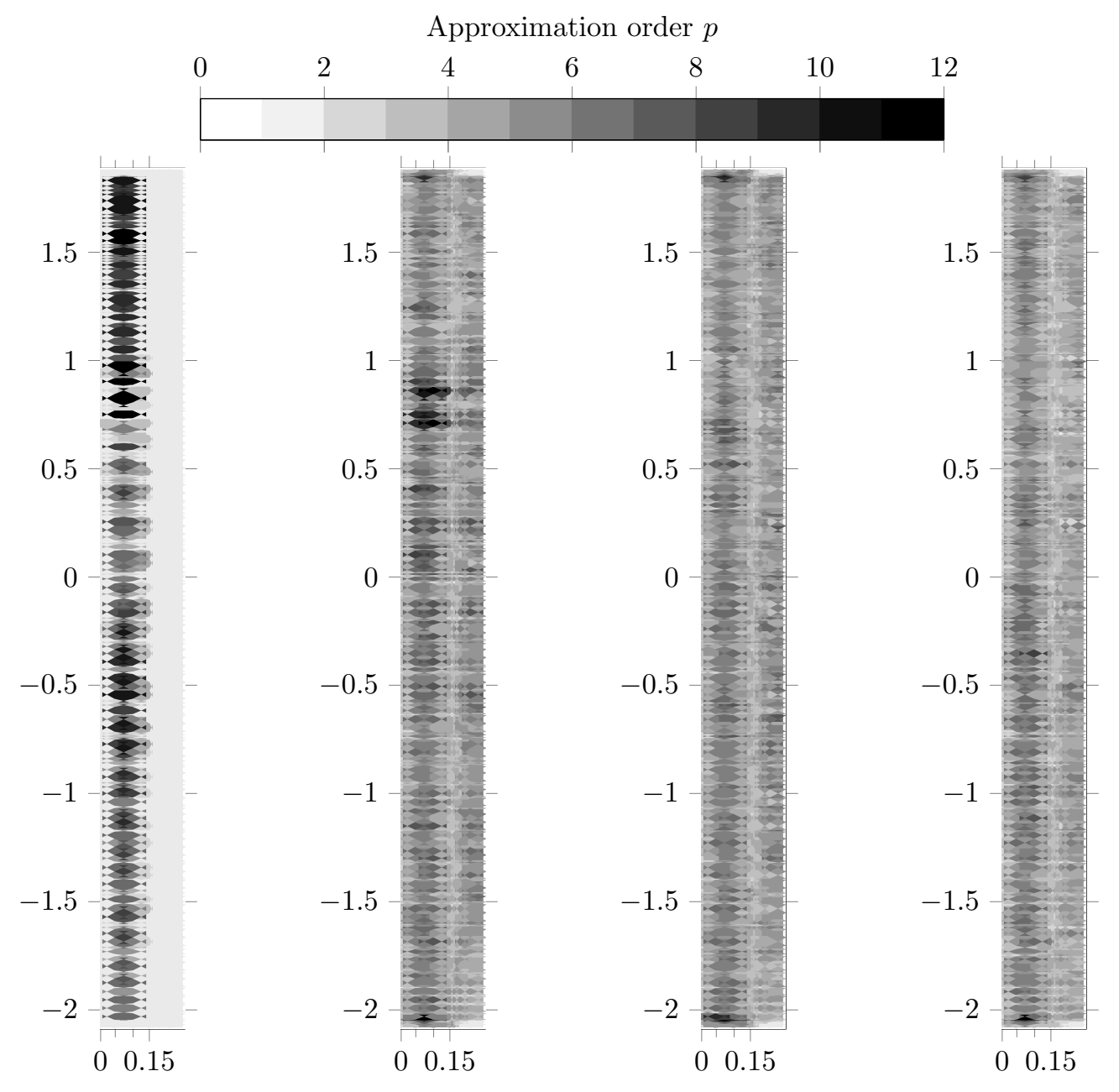

(a) Classical criterion (b) Alternative criterion (c) Classical criterion (d) Alternative criterion without PBI without PBI with PBI with PBI

Figure 21: Final adapted fine meshes after $p$-adaptivity.

[4] Pardo D, Demkowicz L, Torres-Verdín C, Paszynski M. Two-dimensional high-accuracy simulation of resistivity logging-while-drilling (LWD) measurements using a self-adaptive goal-oriented $h p$ finite 
element method. SIAM J. Appl. Math. 2006; 66(6):2085-2106, doi:10.1137/050631732. URL http: //dx.doi.org/10.1137/050631732.

[5] Pardo D, Demkowicz L, Torres-Verdín C, Tabarovsky L. A goal-oriented $h p$-adaptive finite element method with electromagnetic applications. I. Electrostatics. Internat. J. Numer. Methods Engrg. 2006; 65(8):1269-1309, doi:10.1002/nme.1488. URL http://dx.doi.org/10.1002/nme.1488.

[6] Pardo D, Demkowicz L, Torres-Verdín C, Paszynski M. A self-adaptive goal-oriented $h p$ finite element method with electromagnetic applications. II. Electrodynamics. Comput. Methods Appl. Mech. Engrg. 2007; 196(37-40):3585-3597, doi:10.1016/j.cma.2006.10.016. URL http://dx.doi.org/10. $1016 / \mathrm{j} . \mathrm{cma} .2006 \cdot 10.016$.

[7] Pardo D. Multigoal-oriented adaptivity for $h p$-finite element methods. Procedia Computer Science 2010; 1(1):1953 - 1961, doi:http://dx.doi.org/10.1016/j.procs.2010.04.219. URL http://www . sciencedirect.com/science/article/pii/S1877050910002206.

[8] Alvarez-Aramberri J, Pardo D, Barucq H. Inversion of magnetotelluric measurements using multigoal oriented hp-adaptivity. Procedia Computer Science 2013; 18:1564 - 1573, doi:http:// dx.doi.org/10.1016/j.procs.2013.05.324. URL http://www.sciencedirect.com/science/article/ pii/S1877050913004675, 2013 International Conference on Computational Science.

[9] Panetier J, Ladevèze $\mathrm{P}$, Chamoin L. Strict and effective bounds in goal-oriented error estimation applied to fracture mechanics problems solved with XFEM. Internat. J. Numer. Methods Engrg. 2010; 81(6):671-700, doi:10.1002/nme.2705. URL http://dx.doi.org/10.1002/nme.2705.

[10] Waeytens J, Chamoin L, Ladevèze P. Guaranteed error bounds on pointwise quantities of interest for transient viscodynamics problems. Comput. Mech. 2012; 49(3):291-307, doi:10.1007/ s00466-011-0642-1. URL http://dx.doi.org/10.1007/s00466-011-0642-1.

[11] Jhurani C, Demkowicz L. Multiscale modeling using goal-oriented adaptivity and numerical homogenization. Part II: Algorithms for the Moore-Penrose pseudoinverse. Comput. Methods Appl. Mech. Engrg. 2012; 213/216:418-426, doi:10.1016/j.cma.2011.06.003. URL http://dx.doi.org/ $10.1016 / \mathrm{j} . \mathrm{cma} .2011 .06 .003$.

[12] Jhurani C, Demkowicz L. Multiscale modeling using goal-oriented adaptivity and numerical homogenization. Part I: Mathematical formulation and numerical results. Comput. Methods Appl. Mech. Engrg. 2012; 213/216:399-417, doi:10.1016/j.cma.2011.06.011. URL http://dx.doi.org/10.1016/j . cma.2011.06.011.

[13] Verdugo F, Díez P. Computable bounds of functional outputs in linear visco-elastodynamics. Comput. Methods Appl. Mech. Engrg. 2012; 245/246:313-330, doi:10.1016/j.cma.2012.06.016. URL http: //dx.doi.org/10.1016/j.cma.2012.06.016.

[14] Van der Zee KG, Van Brummelen EH, Akkerman I, de Borst R. Goal-oriented error estimation and adaptivity for fluid-structure interaction using exact linearized adjoints. Comput. Methods Appl. Mech. Engrg. 2011; 200(37-40):2738-2757, doi:10.1016/j.cma.2010.12.010. URL http://dx.doi. org/10.1016/j.cma.2010.12.010.

[15] Van der Zee KG, Oden JT, Prudhomme S, Hawkins-Daarud A. Goal-oriented error estimation for Cahn-Hilliard models of binary phase transition. Numer. Methods Partial Differential Equations 2011; 27(1):160-196, doi:10.1002/num.20638. URL http://dx.doi.org/10.1002/num. 20638.

[16] Van der Zee KG, Verhoosel CV. Isogeometric analysis-based goal-oriented error estimation for freeboundary problems. Finite Elem. Anal. Des. 2011; 47(6):600-609, doi:10.1016/j.finel.2010.12.013. URL http://dx.doi.org/10.1016/j.finel.2010.12.013.

[17] Günther A, Hinze M, Tber MH. A posteriori error representations for elliptic optimal control problems with control and state constraints. Constrained optimization and optimal control for partial differential equations, Internat. Ser. Numer. Math., vol. 160. Birkhäuser/Springer Basel AG, Basel, 2012; 303-317, doi:10.1007/978-3-0348-0133-1_17. URL http://dx.doi.org/10.1007/ 978-3-0348-0133-1_17. 
[18] Hintermüller M, Hoppe R, Löbhard C. Dual-weighted goal-oriented adaptive finite elements for optimal control of elliptic variational inequalities. ESAIM: Control, Optimisation and Calculus of Variations 4 2014; 20:524-546, doi:10.1051/cocv/2013074. URL http://www. esaim-cocv.org/article_ S1292811913000742.

[19] Hintermüller M, Hoppe RH. Goal-oriented adaptivity in pointwise state constrained optimal control of partial differential equations. SIAM Journal on Control and Optimization 2010; 48(8):5468-5487, doi:10.1137/090761823. URL http://dx.doi.org/10.1137/090761823.

[20] Pollock S. Convergence of Goal-Oriented Adaptive Finite Element Methods. PhD Thesis 2012. URL http://gateway.proquest.com/openurl?url_ver=Z39.88-2004\&rft_val_fmt= info: of $i / f m t: k e v: m t x:$ dissertation\&res_dat=xri:pqm\&rft_dat=xri:pqdiss:3509792, thesis (Ph.D.)-University of California, San Diego.

[21] Holst M, Pollock S, Zhu Y. Convergence of goal-oriented adaptive finite element methods for semilinear problems. Computing and Visualization in Science 2015; 17(1):43-63, doi:10.1007/ s00791-015-0243-1. URL http://dx.doi.org/10.1007/s00791-015-0243-1.

[22] Holst M, Pollock S. Convergence of goal-oriented adaptive finite element methods for nonsymmetric problems. Numerical Methods for Partial Differential Equations 2016; 32(2):479-509, doi:10.1002/ num.22002. URL http://dx.doi.org/10.1002/num. 22002.

[23] Mommer MS, Stevenson R. A goal-oriented adaptive finite element method with convergence rates. SIAM J. Numer. Anal. 2009; 47(2):861-886, doi:10.1137/060675666. URL http://dx.doi.org/10. $1137 / 060675666$.

[24] Feischl M, Praetorius D, Van der Zee KG. An abstract analysis of optimal goal-oriented adaptivity. SIAM Journal on Numerical Analysis 2016; 54(3):1423-1448, doi:10.1137/15M1021982. URL http: //dx.doi.org/10.1137/15M1021982.

[25] Becker R, Rannacher R. A feed-back approach to error control in finite element methods: basic analysis and examples. East-West J. Numer. Math. 1996; 4(4):237-264.

[26] Becker R, Rannacher R. Weighted a posteriori error control in FE methods. IWR, 1996.

[27] Rannacher R, Suttmeier FT. A posteriori error control in finite element methods via duality techniques: application to perfect plasticity. Comput. Mech. 1998; 21(2):123-133, doi:10.1007/ s004660050288. URL http://dx.doi.org/10.1007/s004660050288.

[28] Paraschivoiu M, Peraire J, Patera AT. A posteriori finite element bounds for linear-functional outputs of elliptic partial differential equations. Comput. Methods Appl. Mech. Engrg. 1997; 150(1-4):289 312, doi:10.1016/S0045-7825(97)00086-8. URL http://dx.doi.org/10.1016/S0045-7825(97) 00086-8, symposium on Advances in Computational Mechanics, Vol. 2 (Austin, TX, 1997).

[29] Paraschivoiu M, Patera AT. A hierarchical duality approach to bounds for the outputs of partial differential equations. Comput. Methods Appl. Mech. Engrg. 1998; 158(3-4):389-407, doi:10.1016/ S0045-7825(99)00270-4. URL http://dx.doi.org/10.1016/S0045-7825(99)00270-4.

[30] Peraire J, Patera AT. Bounds for linear-functional outputs of coercive partial differential equations: local indicators and adaptive refinement. Advances in adaptive computational methods in mechanics (Cachan, 1997), Stud. Appl. Mech., vol. 47. Elsevier Sci. B. V., Amsterdam, 1998; 199-216, doi: 10.1016/S0922-5382(98)80011-1. URL http://dx.doi.org/10.1016/S0922-5382(98)80011-1.

[31] Maday Y, Patera AT, Peraire J. A general formulation for a posteriori bounds for output functionals of partial differential equations; application to the eigenvalue problem. C. R. Acad. Sci. Paris Sér. I Math. 1999; 328(9):823-828, doi:10.1016/S0764-4442(99)80279-1. URL http://dx.doi.org/10. 1016/S0764-4442(99)80279-1.

[32] Peraire J, Patera AT. Asymptotic a posteriori finite element bounds for the outputs of noncoercive problems: the Helmholtz and Burgers equations. Comput. Methods Appl. Mech. Engrg. 1999; 171(1-2):77-86, doi:10.1016/S0045-7825(98)00244-8. URL http://dx.doi .org/10.1016/ S0045-7825 (98) 00244-8. 
[33] Sarrate J, Peraire J, Patera A. A posteriori finite element error bounds for non-linear outputs of the Helmholtz equation. Internat. J. Numer. Methods Fluids 1999; 31(1):17-36, doi:10.1002/ (SICI)1097-0363(19990915)31:1〈17::AID-FLD953〉3.0.CO;2-X. URL http://dx.doi.org/10.1002/ (SICI) 1097-0363(19990915) 31:1<17: :AID-FLD953>3.0.C0;2-X, tenth International Conference on Finite Elements in Fluids (Tucson, AZ, 1998).

[34] Prudhomme S, Oden JT. On goal-oriented error estimation for elliptic problems: application to the control of pointwise errors. Comput. Methods Appl. Mech. Engrg. 1999; 176(1-4):313-331, doi: 10.1016/S0045-7825(98)00343-0. URL http://dx. doi.org/10.1016/S0045-7825(98) 00343-0.

[35] Oden JT, Prudhomme S. New approaches to error estimation and adaptivity for the Stokes and Oseen equations. Internat. J. Numer. Methods Fluids 1999; 31(1):3-15, doi:10.1002/(SICI) 1097-0363(19990915)31:1/3::AID-FLD952〉3.3.CO;2-2. URL http://dx.doi.org/10.1002/(SICI) 1097-0363(19990915) $31: 1<3:$ : AID-FLD952>3 3. CO;2-2, tenth International Conference on Finite Elements in Fluids (Tucson, AZ, 1998).

[36] Oden JT, Prudhomme S. Goal-oriented error estimation and adaptivity for the finite element method. Comput. Math. Appl. 2001; 41(5-6):735-756, doi:10.1016/S0898-1221(00)00317-5. URL http://dx. doi.org/10.1016/S0898-1221(00)00317-5.

[37] Prudhomme S, Oden JT. Computable error estimators and adaptive techniques for fluid flow problems. Error estimation and adaptive discretization methods in computational fluid dynamics, Lect. Notes Comput. Sci. Eng., vol. 25. Springer, Berlin, 2003; 207-268, doi:10.1007/978-3-662-05189-4_5. URL http://dx.doi.org/10.1007/978-3-662-05189-4_5.

[38] Darrigrand V, Pardo D, Muga I. Goal-oriented adaptivity using unconventional error representations for the 1D Helmholtz equation. Computers \& Mathematics with Applications 2015; 69(9):964 - 979, doi:http://dx.doi.org/10.1016/j.camwa.2015.03.006. URL http://www. sciencedirect.com/ science/article/pii/S0898122115001017.

[39] Cao W, Demkowicz L. Optimal error estimate of a projection based interpolation for the $p$-version approximation in three dimensions. Computers $\& 3$ Mathematics with Applications 2005; 50(3):359 366 .

[40] Demkowicz L. Computing with hp-adaptive finite elements. Vol. 1. One and two dimensional elliptic and Maxwell problems. Chapman \& Hall/CRC Applied Mathematics and Nonlinear Science Series, Chapman \& Hall/CRC, Boca Raton, FL, 2007, doi:10.1201/9781420011692. URL http://dx.doi . org/10.1201/9781420011692.

[41] Demkowicz L, Kurtz J, Pardo D, Paszyński M, Rachowicz W, Zdunek A. Computing with hp-adaptive finite elements. Vol. 2. Frontiers: three dimensional elliptic and Maxwell problems with applications. Applied Mathematics and Nonlinear Science Series, Chapman \& Hall/CRC, Boca Raton, FL, 2008.

[42] Ihlenburg F, Babuška I. Finite element solution of the Helmholtz equation with high wave number. I. The $h$-version of the FEM. Comput. Math. Appl. 1995; 30(9):9-37, doi:10.1016/0898-1221(95) 00144-N. URL http://dx.doi.org/10.1016/0898-1221(95)00144-N.

[43] Ihlenburg F, Babuška I. Dispersion analysis and error estimation of Galerkin finite element methods for the Helmholtz equation. Internat. J. Numer. Methods Engrg. 1995; 38(22):3745-3774, doi:10. 1002/nme.1620382203. URL http://dx.doi.org/10.1002/nme.1620382203.

[44] Babuška I, Ihlenburg F, Strouboulis T, Gangaraj SK. A posteriori error estimation for finite element solutions of Helmholtz' equation. I. The quality of local indicators and estimators. Internat. $\mathrm{J}$. Numer. Methods Engrg. 1997; 40(18):3443-3462, doi:10.1002/(SICI)1097-0207(19970930)40:18<3443:: AID-NME221)3.3.CO;2-T. URL http://dx.doi.org/10.1002/(SICI) 1097-0207(19970930)40: 18<3443: : AID-NME221>3.3.CO;2-T.

[45] Babuška I, Ihlenburg F, Strouboulis T, Gangaraj SK. A posteriori error estimation for finite element solutions of Helmholtz' equation. II. Estimation of the pollution error. Internat. $\mathrm{J} . \mathrm{Nu}-$ mer. Methods Engrg. 1997; 40(21):3883-3900, doi:10.1002/(SICI)1097-0207(19971115)40:21/3883:: AID-NME231>3.0.CO;2-V. URL http://dx.doi.org/10.1002/(SICI) 1097-0207(19971115)40: 21<3883: : AID-NME231>3.0.CO;2-V. 
[46] Ihlenburg F, Babuška I. Finite element solution of the Helmholtz equation with high wave number. II. The $h-p$ version of the FEM. SIAM J. Numer. Anal. 1997; 34(1):315-358, doi:10.1137/ S0036142994272337. URL http://dx.doi.org/10.1137/S0036142994272337.

[47] Babuška IM, Sauter SA. Is the pollution effect of the FEM avoidable for the Helmholtz equation considering high wave numbers? SIAM Rev. 2000; 42(3):451-484 (electronic), doi:10.1137/ S0036142994269186. URL http://dx.doi.org/10.1137/S0036142994269186, reprint of SIAM J. Numer. Anal. 34 (1997), no. 6, 2392-2423 [ MR1480387 (99b:65135)].

[48] Steffens LM, Parés N, Díez P. Estimation of the dispersion error in the numerical wave number of standard and stabilized finite element approximations of the Helmholtz equation. Internat. $J$. Numer. Methods Engrg. 2011; 86(10):1197-1224, doi:10.1002/nme.3104. URL http://dx.doi.org/ 10.1002/nme. 3104 .

[49] Steffens LM, Parés N, Díez P. Goal-oriented $h$-adaptivity for the Helmholtz equation: error estimates, local indicators and refinement strategies. Comput. Mech. 2011; 47(6):681-699, doi: 10.1007/s00466-010-0557-2. URL http://dx.doi.org/10.1007/s00466-010-0557-2.

[50] Steffens LM, Díez P. A simple strategy to assess the error in the numerical wave number of the finite element solution of the Helmholtz equation. Comput. Methods Appl. Mech. Engrg. 2009; 198(15-16):1389-1400, doi:10.1016/j.cma.2008.12.005. URL http://dx.doi.org/10.1016/j.cma. 2008.12.005.

[51] Van der Zee KG. Goal-adaptive discretization of fluid-structure interaction. PhD Thesis, Delft University of Technology, http://repository.tudelft.nl June 2009.

[52] Babuška I, Rheinboldt WC. Error estimates for adaptive finite element computations. SIAM Journal on Numerical Analysis 1978; 15(4):736-754, doi:10.1137/0715049. URL http://dx.doi.org/10. $1137 / 0715049$.

[53] Becker R, Rannacher R. An optimal control approach to a posteriori error estimation in finite element methods. Acta Numer. 2001; 10:1-102, doi:10.1017/S0962492901000010. URL http://dx.doi.org/ $10.1017 /$ S0962492901000010.

[54] Giles MB, Süli E. Adjoint methods for PDEs: a posteriori error analysis and postprocessing by duality. Acta Numer. 2002; 11:145-236, doi:10.1017/S096249290200003X. URL http://dx.doi. org/10.1017/S096249290200003X.

[55] Gaspoz FD, Morin P. Convergence rates for adaptive finite elements. IMA J. Numer. Anal. 2009; 29(4):917-936, doi:10.1093/imanum/drn039. URL http://dx.doi.org/10.1093/imanum/drn039.

[56] Balay S, Abhyankar S, Adams MF, Brown J, Brune P, Buschelman K, Dalcin L, Eijkhout V, Gropp WD, Kaushik D, et al.. PETSc Web page. http://www.mcs.anl.gov/petsc 2015. URL http:// www.mcs.anl.gov/petsc.

[57] Demkowicz L, Babuška I. $p$ interpolation error estimates for edge finite elements of variable order in two dimensions. SIAM J. Numer. Anal. 2003; 41(4):1195-1208, doi:10.1137/S0036142901387932. URL http://dx.doi.org/10.1137/S0036142901387932.

[58] Oden JT, Prudhomme S, Demkowicz L. A posteriori error estimation for acoustic wave propagation problems. Arch. Comput. Methods Engrg. 2005; 12(4):343-389, doi:10.1007/BF02736190. URL http: //dx.doi.org/10.1007/BF02736190.

[59] Ainsworth M. Discrete dispersion relation for $h p$-version finite element approximation at high wave number. SIAM J. Numer. Anal. 2004; 42(2):553-575, doi:10.1137/S0036142903423460. URL http: //dx.doi.org/10.1137/S0036142903423460.

[60] Romkes A, Oden J. Adaptive modeling of wave propagation in heterogeneous elastic solids. Computer Methods in Applied Mechanics and Engineering 2004; 193(6-8):539 - 559, doi:http: //doi.org/10.1016/j.cma.2003.10.014. URL http://www.sciencedirect.com/science/article/ $\mathrm{pii} / \mathrm{S} 0045782503005772$.

[61] Collier N, Radwan H, Dalcin L, Calo VM. Time adaptivity in the diffusive wave approximation to the shallow water equations. Journal of Computational Science 2013; 4(3):152-156. 
[62] Verdugo F, Parés N, Díez P. Goal-oriented space-time adaptivity for transient dynamics using a modal description of the adjoint solution. Comput. Mech. 2014; 54(2):331-352, doi:10.1007/ s00466-014-0988-2. URL http://dx.doi.org/10.1007/s00466-014-0988-2.

[63] Pardo D, Matuszyk P, Muga I, Torres-Verdín C, Mora A, Calo VM. Simulation of wireline sonic logging measurements acquired with borehole-eccentered tools using a high-order adaptive finite-element method. Journal of Computational Physics 2011; 230(16):6320 - 6333, doi:http: //dx.doi.org/10.1016/j.jcp.2011.04.028. URL http://www.sciencedirect.com/science/article/ $\mathrm{pii/S0021999111002762.}$

[64] Matuszyk PJ, Torres-Verdín C, Pardo D. Frequency-domain finite-element simulations of 2d sonic wireline borehole measurements acquired in fractured and thinly bedded formations. GEOPHYSICS 2013; 78(4):D193-D207, doi:10.1190/geo2012-0397.1. URL http://dx.doi.org/10. 1190/geo2012-0397.1.

[65] Matuszyk PJ, Torres-Verdín C. Frequency-domain simulation of logging-while-drilling borehole sonic waveforms. GEOPHYSICS 2014; 79(2):D99-D113, doi:10.1190/geo2013-0279.1. URL http://dx. doi.org/10.1190/geo2013-0279.1.

[66] Alvarez-Aramberri J, Pardo D, Barucq H. A secondary field based $h p$-Finite Element Method for the simulation of magnetotelluric measurements. Journal of Computational Science 11 2015; 11:137-144, doi:http://doi.org/10.1016/j.jocs.2015.02.005. URL http://www.sciencedirect.com/ science/article/pii/S1877750315000150. 